my journal manuscript No.

(will be inserted by the editor)

\title{
Error Analysis of Modified Langevin Dynamics
}

\author{
Stephane Redon ${ }^{\dagger}$. Gabriel Stoltz ${ }^{\ddagger}$. \\ Zofia Trstanova ${ }^{\dagger}$
}

Received: date / Accepted: date

\begin{abstract}
We consider Langevin dynamics associated with a modified kinetic energy vanishing for small momenta. This allows us to freeze slow particles, and hence avoid the re-computation of inter-particle forces, which leads to computational gains. On the other hand, the statistical error may increase since there are a priori more correlations in time. The aim of this work is first to prove the ergodicity of the modified Langevin dynamics (which fails to be hypoelliptic), and next to analyze how the asymptotic variance on ergodic averages depends on the parameters of the modified kinetic energy. Numerical results illustrate the approach, both for low-dimensional systems where we resort to a Galerkin approximation of the generator, and for more realistic systems using Monte Carlo simulations.
\end{abstract}

Keywords Langevin dynamics · Variance reduction · Ergodicity · Functional estimates $\cdot$ Linear response

\section{Introduction}

A fundamental purpose of molecular simulation is the computation of macroscopic quantities, typically through averages of functions of the variables of the system with respect to a given probability measure $\mu$, which defines the macroscopic state of the system. In the most common setting, the probability measure $\mu$ with respect to which averages are computed corresponds to the canonical ensemble (see for instance Tuckerman (2010)). Its distribution is defined by the Boltzmann-Gibbs density, which models the configurations of a conservative system in contact with a heat bath at fixed

\footnotetext{
$\dagger$

Inria - Univ. Grenoble Alpes, LJK, F-38000 Grenoble, France

CNRS, LJK, F-38000 Grenoble, France

E-mail: zofia.trstanova@inria.fr

$\ddagger$

Université Paris-Est, CERMICS (ENPC), INRIA, F-77455 Marne-la-Vallée, France
} 
temperature. Numerically, high-dimensional averages with respect to $\mu$ are often approximated as ergodic averages over realizations of appropriate stochastic differential equations (SDEs):

$$
\lim _{t \rightarrow \infty} \hat{A}_{t}=\mathbb{E}_{\mu}(A) \quad \text { a.s., } \quad \hat{A}_{t}:=\frac{1}{t} \int_{0}^{t} A\left(p_{s}, q_{s}\right) d s .
$$

A typical dynamics to this end is the Langevin dynamics

$$
\left\{\begin{array}{l}
d q_{t}=M^{-1} p_{t} d t \\
d p_{t}=-\nabla V\left(q_{t}\right) d t-\gamma M^{-1} p_{t} d t+\sqrt{\frac{2 \gamma}{\beta}} d W_{t},
\end{array}\right.
$$

where $d W_{t}$ is a standard Wiener process, $V$ the potential energy function, $\gamma>0$ a friction coefficient, $M$ the mass matrix of the system, and $\beta$ is proportional to the inverse temperature (see Section 2 for more precise definitions). For references on the ergodicity of Langevin dynamics, we refer the reader to Talay (2002) and Mattingly et al. (2002), for instance.

There are two main sources of error in the computation of average properties such as $\mathbb{E}_{\mu}(A)$ through time averages as in (1): (i) a systematic bias (or perfect sampling bias) related to the use of a discretization method for the SDEs (and usually proportional to a power of the integration step size $\Delta t$ ), and (ii) statistical errors, due to the finite lengths of the sampling paths involved and the underlying variance of the random variables. The first point was studied in Leimkuhler et al. (2015) for standard Langevin dynamics. Our focus in this work is on the statistical error.

Statistical errors may be large when the dynamics is metastable, i.e. when the system remains trapped for a very long time in some region of the configuration space (called a metastable region) before hopping to another metastable region. Metastability implies that the convergence of averages over trajectories is very slow, and that transitions between metastable regions (which are typically the events of interest at the macroscopic level) are very rare. In fact, metastability arises from the multimodality of the probability measure sampled by the dynamics. We refer for instance to Lelièvre (2013) for a review on ways to quantify the metastability of sampling dynamics. There are various strategies to reduce the variance of time averages by reducing the metastability. The most famous one is importance sampling: the potential energy function $V$ is modified by an additional term $V$ so that the Langevin dynamics associated with $V+\widetilde{V}$ is less metastable. An automatic way of doing so is to consider a so-called reaction coordinate, and define $\widetilde{V}$ as the opposite of the associated free energy (see Lelièvre et al. (2010); Lelièvre and Stoltz (2015) for further precisions).

We explore here an alternative path, which consists in modifying the kinetic energy rather than the potential energy. Indeed, recall that the difficult part in sampling the canonical measure is in sampling positions (see Section 2 for a more precise discussion of this point). There is therefore some freedom in the choice of the kinetic energy if the goal is to compute average properties.

Previous works in this direction focused on changing the mass matrix in order to increase the time steps used in the simulation (see e.g. Bennett (1975); Plechac and Rousset (2010)). The mathematical analysis we provide is inspired by a recent 
work by Artemova and Redon (2012) where the kinetic energy of each particle is more drastically modified: it is set to 0 when the particle's momenta are small, while it remains unchanged for larger momenta. In such adaptively restrained (AR) simulations, particles may become temporarily frozen, while their momenta may continue to evolve. Since, in many cases, inter-particle forces only depend on relative particle positions, and hence do not have to be updated when particles are frozen, adaptively restrained particle simulations may yield a significant algorithmic speedup $S_{\text {algo }}$ when a sufficiently large number of particles are frozen at each time step (or, more generally, when inter-particle distances remain constant and particle forces are expressed in local reference frames). This has been demonstrated in several contexts, e.g. for modeling hydrocarbon systems (Bosson et al. (2012)), proteins (Rossi et al. (2007)), and for electronic structure calculations (Bosson et al. (2013)).

Unfortunately, freezing particles even temporarily may make iterates more correlated, which may translate into an increase of the statistical error $\sigma_{\text {mod }}^{2}$ observed for modified Langevin dynamics, compared to the statistical error $\sigma_{\text {std }}^{2}$ observed for standard Langevin dynamics. The actual speed-up of the method, in terms of the total wall-clock time needed to achieve a given precision in the estimation of an observable, should therefore be expressed as:

$$
S_{\text {actual }}=S_{\text {algo }} \frac{\sigma_{\text {std }}^{2}}{\sigma_{\text {mod }}^{2}} .
$$

Our aim here is thus to quantify the increase in the variance as a function of the parameters of the modified kinetic energy. In fact, a first task is to prove that the Langevin dynamics with modified kinetic energy is indeed ergodic, and that the variance is well defined. This is unclear at first sight since the modified dynamics fails to be hypoelliptic (see the discussion in Section 3.1).

This article is organized as follows. In Section 2, we introduce the modified Langevin dynamics we consider, and present the particular case of the AR-Langevin dynamic. The ergodicity of these dynamics is proved in Section 3, both in terms of almost-sure convergence of time averages along a single realization, and in terms of the law of the process. We also provide a result on the regularity of the evolution semi-group, adapted from similar estimates for standard Langevin dynamics in Talay (2002). Such estimates allow us to analyze the statistical error in Section 4. We state in particular a Central Limit Theorem for $\widehat{A}_{t}$, and perform a perturbative study of the asymptotic variance of the AR-Langevin dynamics in some limiting regime. Our theoretical findings are illustrated by numerical simulations in Section 5, both in a simple one-dimensional case where the variance can be accurately computed using an appropriate Galerkin approximation, as well as for a more realistic system for which we resort to Monte-Carlo simulations. The proofs of our results are gathered in Section 6.

\section{Modified Langevin dynamics}

We consider a system of $N$ particles in spatial dimension $D$, so that the total dimension of the system is $d:=D \times N$. The vectors of positions and momenta are denoted 
respectively by $q=\left(q_{1}, \cdots, q_{N}\right)$ and $p=\left(p_{1}, \cdots, p_{N}\right)$. Periodic boundary conditions are used for positions, so that the phase-space of admissible configurations is $\mathscr{E}=$ $\mathscr{D} \times \mathbb{R}^{d}$ with $\mathscr{D}:=(L \mathbb{T})^{d}, \mathbb{T}=\mathbb{R} \backslash \mathbb{Z}$ being the one-dimensional unit torus and $L>0$ the size of the simulation box.

In order to possibly increase the rate of convergence of the ergodic averages (1), we modify the Langevin dynamics (2) by changing the kinetic energy. More precisely, instead of the standard quadratic kinetic energy

$$
U_{\text {std }}(p)=\frac{1}{2} p^{T} M^{-1} p, \quad M=\operatorname{diag}\left(m_{1}, \ldots, m_{N}\right),
$$

we introduce a general kinetic energy function $U: \mathbb{R}^{d} \rightarrow \mathbb{R}$. The total energy of the system is then characterized by the Hamiltonian

$$
H(p, q)=U(p)+V(q) .
$$

In order to ensure that the measure $\mathrm{e}^{-\beta H(q, p)} d q d p$ can be normalized, and in order to simplify the mathematical analysis, we make in the sequel the following assumption.

Assumption 1 The potential energy function $V$ belongs to $C^{\infty}(\mathscr{D}, \mathbb{R})$, and $U \in C^{\infty}\left(\mathbb{R}^{d}, \mathbb{R}\right)$ grows sufficiently fast at infinity in order to ensure that $\mathrm{e}^{-\beta U} \in L^{1}\left(\mathbb{R}^{d}\right)$.

The Langevin dynamics associated with a general Hamiltonian reads

$$
\left\{\begin{array}{l}
d q_{t}=\nabla_{p} H\left(p_{t}, q_{t}\right) d t \\
d p_{t}=-\nabla_{q} H\left(p_{t}, q_{t}\right) d t-\gamma \nabla_{p} H\left(p_{t}, q_{t}\right) d t+\sqrt{\frac{2 \gamma}{\beta}} d W_{t},
\end{array}\right.
$$

where $d W_{t}$ is a standard $d$-dimensional Wiener process and $\gamma>0$ is the friction constant. For the separable Hamiltonian (4), the general Langevin dynamics simplifies as

$$
\left\{\begin{array}{l}
d q_{t}=\nabla U\left(p_{t}\right) d t \\
d p_{t}=-\nabla V\left(q_{t}\right) d t-\gamma \nabla U\left(p_{t}\right) d t+\sqrt{\frac{2 \gamma}{\beta}} d W_{t} .
\end{array}\right.
$$

The generator of the process (5) reads

$$
\mathscr{L}=\nabla U \cdot \nabla_{q}-\nabla V \cdot \nabla_{p}+\gamma\left(-\nabla U \cdot \nabla_{p}+\frac{1}{\beta} \Delta_{p}\right) .
$$

A simple computation shows that the canonical distribution

$$
\mu(d q d p)=Z_{\mu}^{-1} \mathrm{e}^{-\beta H(q, p)} d p d q, \quad Z_{\mu}=\int_{\mathscr{E}} \mathrm{e}^{-\beta H(q, p)} d p d q<+\infty,
$$

is invariant under the dynamics (5), i.e. for all $C^{\infty}$ functions $\phi$ with compact support,

$$
\int_{\mathscr{E}} \mathscr{L} \phi d \mu=0
$$


Note that, in view of the separability of the Hamiltonian, the marginal of the distribution $\mu$ in the position variables is, for any kinetic energy $U$,

$$
\bar{\mu}(d q)=Z_{V}^{-1} \mathrm{e}^{-\beta V(q)} d q, \quad Z_{V}=\int_{\mathscr{D}} \mathrm{e}^{-\beta V(q)} d q .
$$

In particular, this marginal distribution therefore coincides with the one of the standard Langevin dynamics (2). This allows to straightforwardly estimate canonical averages of observables depending only on the positions with the modified Langevin dynamics (5). In fact, there is no restriction in generality in considering observables depending only on the positions, since general observables $A(q, p)$ depending both on momenta and positions can be reduced to functions of the positions only by a partial integration in the momenta variables. This partial integration is often very easy to perform since momenta are independent Gaussian random variables under the canonical measure associated with the standard kinetic energy.

\subsection{AR-Langevin dynamics}

A concrete example for the choice of the kinetic energy function $U$ in (4) is the one proposed for the adaptively restrained Langevin dynamics in Artemova and Redon (2012). It is parameterized by two constants $0 \leqslant K_{\min }<K_{\max }$. In this model, the kinetic energy is a sum of individual contributions

$$
U(p)=\sum_{i=1}^{N} u\left(p_{i}\right)
$$

For large values of momenta, the modified individual kinetic energies are equal to the standard kinetic energy of one particle, but they vanish for small momenta:

$$
u\left(p_{i}\right)=\left\{\begin{array}{cl}
0 & \text { for } \frac{p_{i}^{2}}{2 m_{i}} \leqslant K_{\min }, \\
\frac{p_{i}^{2}}{2 m_{i}} & \text { for } \frac{p_{i}^{2}}{2 m_{i}} \geqslant K_{\max } .
\end{array}\right.
$$

An appropriate function allows to smoothly interpolate between these two limiting regimes (see Definition 1 for the precise expression). A possible choice of an individual kinetic energy $u$, as well as the associated canonical distribution of momenta $Z_{u}^{-1} \mathrm{e}^{-\beta u(p)} d p$ are depicted in Figure 1 and Figure 2 when $D=1$.

The interest of AR-Langevin dynamics is that, when their individual kinetic energies are sufficiently small, particles do not move. When two particles are frozen in this way, their pairwise interactions need not be updated. This allows decreasing the computational complexity of the force computation, which is typically the most time-consuming part of a molecular dynamics solver. Note that this can be generalized to higher-order interactions (such as three-body interactions based on bending angles for instance). 


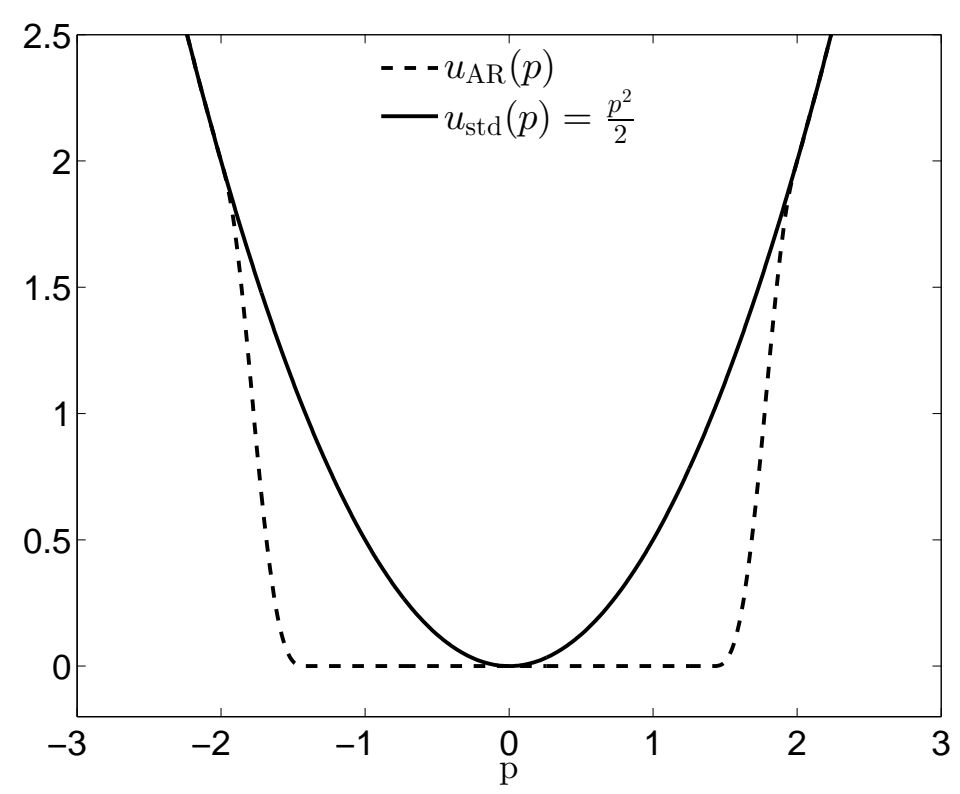

Fig. 1: Standard quadratic kinetic energy function $U_{\text {std }}$ (solid lines), and an example of an AR kinetic energy function $u$ with parameters $K_{\max }=2$ and $K_{\min }=1$ (dashed line).

Remark 1 Note that, due to the additive structure of the kinetic energy, the momenta $p_{i}$ are independent and identically distributed (i.i.d.) under the canonical measure. It is however possible to choose different parameters $K_{\min }$ and $K_{\max }$ for different particles, for example to focus calculations on a specific part of the particle system, in which case the momenta are still independent but not longer identically distributed. Such a situation is considered in the numerical example presented in Section 5.2.

\section{Ergodicity of the modified Langevin dynamics}

There are several notions of ergodicity for stochastic processes. We focus here on two of them: the convergence of ergodic averages over a single trajectory, and the convergence of the law of the process.

\subsection{Convergence of ergodic averages}

The convergence of ergodic averages over one trajectory is automatically ensured by the existence of an invariant probability measure and the irreducibility of the dynamics (see for instance Kliemann (1987); Meyn and Tweedie (1993) for early results on such convergences for possibly degenerate diffusions). Since, by construction, an invariant probability measure is known (namely the canonical measure (7)), it suffices 


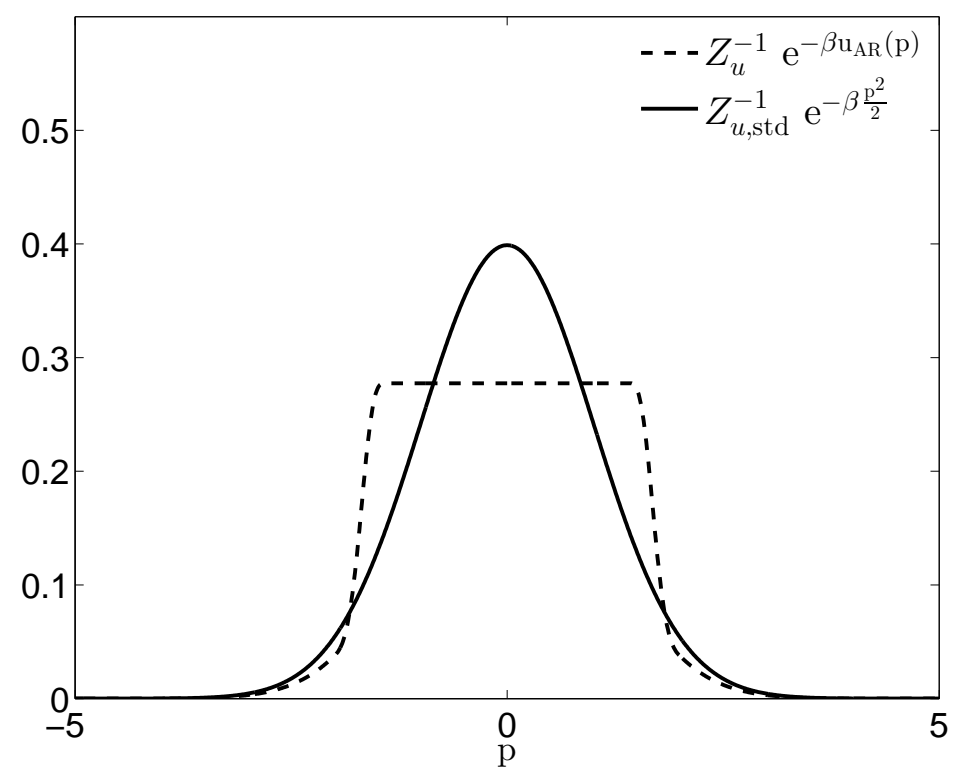

Fig. 2: Marginal canonical densities associated with the kinetic energy functions of Figure 1.

to show that the process generated by the modified Langevin equation is irreducible to conclude to the convergence of ergodic averages.

As reviewed in Rey-Bellet (2006), the most standard argument to prove the irreducibility of degenerate diffusions is to prove the controllability of the dynamics relying on the Stroock-Varadhan support theorem, and the regularity of the transition kernel thanks to some hypoellipticity property. These conditions are satisfied for standard Langevin dynamics (see for instance Mattingly et al. (2002)), but not for the modified Langevin dynamics we consider, since the Hessian of the kinetic energy function may not be invertible on an open set. This is the case for the AR kinetic energy function presented in Section 2.1.

To illustrate this point, let us show for instance how the standard way of proving hypoellipticity fails (the proof of the controllability faces similar issues). The first task is to rewrite the generator (6) of the process as

$$
\mathscr{L}=X_{0}-\sum_{j=1}^{d} X_{j}^{\dagger} X_{j}
$$

where

$$
X_{0}=\nabla U \cdot \nabla_{q}-\nabla V \cdot \nabla_{p}-\gamma \nabla U \cdot \nabla_{p}, \quad X_{j}=\sqrt{\frac{\gamma}{\beta}} \partial_{p_{j}}
$$


and $X_{j}^{\dagger}$ is the adjoint of $X_{j}$ on the flat space $L^{2}(\mathscr{E})$. We next compute, for $j=1, \ldots, d$, the commutators

$$
\left[X_{0}, X_{j}\right]=X_{0} X_{j}-X_{j} X_{0}=\sqrt{\frac{\gamma}{\beta}} \nabla\left(\partial_{p_{j}} U\right) \cdot\left(\nabla_{q}-\gamma \nabla_{p}\right) .
$$

When $\nabla^{2} U$ is invertible, it is possible to recover the full algebra of derivatives by an appropriate combination of $X_{1}, \ldots, X_{d}$ and $\left[X_{0}, X_{1}\right], \ldots,\left[X_{0}, X_{d}\right]$. Here, we consider a situation when this is not the case and, even more dramatically, where the Hessian may vanish on an open set. In this situation, $\left[X_{0}, X_{j}\right]=0$ on the same open set, and in fact all iterated commutators $\left[X_{0},\left[\ldots\left[X_{0}, X_{j}\right]\right]\right]$ also vanish.

We solve this problem by a direct constructive approach, where we see the modified dynamics as a perturbation of the standard Langevin dynamics. We rely on the following assumption:

Assumption 2 The kinetic energy function $U \in C^{\infty}$ of the modified Langevin dynamics is such that

$$
\left\|\nabla U-\nabla U_{\text {std }}\right\|_{L^{\infty}} \leqslant G_{\text {std }}
$$

for some constant $G_{\text {std }}<+\infty$.

Under this assumption, we can prove that the modified Langevin dynamics is irreducible by proving an appropriate minorization condition, which crucially relies on the compactness of the position space $\mathscr{D}$ (see Section 6.2 for the proof).

Lemma 1 (Minorization condition) Suppose Assumption 2 holds. Then for any fixed $p^{*}>0$ and $t>0$, there exists a probability measure $v_{p^{*}, t}$ on $\mathscr{D} \times \mathbb{R}^{d}$ and a constant $\kappa>0$ such that, for every Borel set $B \in \mathscr{B}(\mathscr{E})$,

$$
\mathbb{P}\left(\left(q_{t}, p_{t}\right) \in B|| p_{0} \mid \leqslant p_{*}\right) \geqslant \kappa v_{p_{*}, t}(B),
$$

with $v_{p_{*}, t}(B)>0$ when $|B|>0$.

The minorization condition implies the irreducibility of the dynamics, so that the following convergence result readily follows.

Theorem 3 (Convergence of ergodic averages) When Assumption 2 holds, ergodic averages over trajectories almost surely converge to the canonical average:

$$
\forall A \in L^{1}(\mu), \quad \lim _{t \rightarrow+\infty} \frac{1}{t} \int_{0}^{t} A\left(q_{s}, p_{s}\right) d s=\int_{\mathscr{E}} A d \mu \quad \text { a.s. }
$$

\subsection{Convergence of the law}

There are various functional frameworks to measure the convergence of the law of the process. We consider here weighted $L^{\infty}$ estimates on the semi-group $\mathrm{e}^{t \mathscr{L}}$. More precisely, we introduce a scale of Lyapunov functions

$$
\mathscr{K}_{s}(p):=1+|p|^{2 s}
$$


for $s \in \mathbb{N}^{*}$. Recall indeed that only momenta need to be controlled since positions remain in a compact space. The associated weighted $L^{\infty}$ spaces are

$$
L_{\mathscr{K}_{s}}^{\infty}=\left\{f \text { measurable } \mid\|f\|_{L_{\mathscr{K}_{s}}^{\infty}}:=\left\|\frac{f}{\mathscr{K}_{s}}\right\|_{L^{\infty}}<+\infty\right\} .
$$

In order to prove the exponential convergence of the law, we rely on the result of Hairer and Mattingly (2011), which states that if a Lyapunov condition and a minorization condition hold true, then the sampled chain converges exponentially fast to its steady state in the following sense.

Theorem 4 (Exponential convergence of the law) Suppose that Assumption 2 holds. Then the invariant measure $\mu$ is unique, and for any $s \in \mathbb{N}^{*}$, there exist constants $C_{s}, \lambda_{s}>0$ such that

$$
\forall f \in L_{\mathscr{K}_{s}}^{\infty}, \quad \forall t \geqslant 0, \quad\left\|\mathrm{e}^{t \mathscr{L}} f-\int_{\mathscr{E}} f d \mu\right\|_{L_{\mathscr{K}_{s}}^{\infty}} \leqslant C_{s} \mathrm{e}^{-\lambda_{s} t}\|f\|_{L_{\mathscr{K}_{s}}^{\infty}} .
$$

As mentioned above, the proof of this result directly follows from the arguments of Hairer and Mattingly (2011). The minorization condition is already stated in Lemma 1, while the appropriate Lyapunov condition reads as follows (see Section 6.1 for the proof, which uses the same strategy as Leimkuhler et al. (2015) and Joubaud et al. (2015)).

Lemma 2 (Lyapunov Condition) Suppose that Assumption 2 holds. Then, for any $s \geqslant 1$ and $t>0$, there exist $b>0$ and $a \in[0,1)$ such that

$$
\mathrm{e}^{t \mathscr{L}} \mathscr{K}_{s} \leqslant a \mathscr{K}_{s}+b
$$

\subsection{Regularity results for the evolution semi-group}

We provide in this section decay estimates for the spatial derivatives of $\mathrm{e}^{t \mathscr{L}} f$, following the approach pioneered in Talay (2002) and further refined in Kopec (2013). Such estimates were obtained for the standard Langevin dynamics, but can in fact straightforwardly be extended to modified Langevin dynamics with Hessians bounded from below by a positive constant. Our aim in this section is to provide decay estimates for the spatial derivatives of $\mathrm{e}^{t \mathscr{L}} f$ in the situation when $\nabla^{2} U$ fails to be strictly convex, for instance because $\nabla^{2} U$ vanishes on an open set as is the case for AR particle simulations.

In order to state our results, we first need to define the weighted Sobolev spaces $\mathscr{W}_{\mathscr{K}_{s}}^{n, \infty}$ for $n \in \mathbb{N}$ :

$$
\mathscr{W}_{\mathscr{K}_{s}}^{n, \infty}=\left\{f \in L_{\mathscr{K}_{s}}^{\infty}\left|\forall k \in \mathbb{N}^{2 d},\right| k \mid \leqslant n, \partial^{k} f \in L_{\mathscr{K}_{s}}^{\infty}\right\} .
$$

These spaces gather all functions which grow at most like $\mathscr{K}_{s}$, and whose derivatives of order at most $n$ all grow at most like $\mathscr{K}_{s}$. We also introduce the space of smooth 
functions $\mathscr{S}$, the vector space of functions $f \in L^{2}(\mu)$ such that, for any $n \geqslant 0$, there exists $r \in \mathbb{N}$ for which $f \in \mathscr{W}_{\mathscr{K}_{r}}^{n, \infty}$.

We also make the following assumption on the kinetic energy function, which can be understood as a condition of "almost strict convexity" of the Hessian $\nabla^{2} U$.

Assumption 5 The kinetic energy $U \in \mathscr{S}$ has bounded second-order derivatives:

$$
\sup _{|j|=2}\left\|\partial^{j} U\right\|_{L^{\infty}}<\infty
$$

and there exist a function $U_{v} \in \mathscr{S}$ and constants $v>0$ and $G_{v} \geqslant 0$ such that

$$
\nabla^{2} U_{v} \geqslant v>0
$$

and

$$
\left\|\nabla\left(U-U_{v}\right)\right\|_{L^{\infty}} \leqslant G_{V} .
$$

Remark 2 A natural choice for the function $U_{v}$ in Assumption 5 is $U_{\text {std }}$. The condition (11) then holds with $v=1 / \max \left(m_{1}, \ldots, m_{N}\right)$. Moreover, (10) holds true as soon as $U$ is a local perturbation of $U_{\text {std. }}$. The most demanding condition therefore is (12), especially if $G_{V}$ has to be small.

By following the same strategy as in (Kopec 2013, Proposition A.1.) (which refines the results already obtained in Talay (2002)), and appropriately taking care of the lack of strict positivity of the Hessian $\nabla^{2} U$ by assuming that $G_{v}$ is sufficiently small, we prove the following result in Section 6.3.

Lemma 3 Suppose that Assumptions 2 and 5 hold, and fix $A \in \mathscr{S}$. For any $n \geqslant$ 1 , there exist $\widetilde{n}, s_{n} \in \mathbb{N}$ and $\lambda_{n}>0$ such that, for $s \geqslant s_{n}$ and $G_{v} \leqslant \rho_{s}$ with $\rho_{s}>0$ sufficiently small (depending on s but not on $n$ ), there is $r \in \mathbb{N}$ and $C>0$ for which

$$
\forall t \geqslant 0, \quad \forall|k| \leqslant n, \quad\left\|\partial^{k} \mathrm{e}^{t \mathscr{L}} \Pi_{\mu} A\right\|_{L_{\mathscr{K}_{s}}^{\infty}} \leqslant C\|A\|_{W_{\mathscr{\mathscr { K } _ { r }}}^{\tilde{n}, \infty}} \mathrm{e}^{-\lambda_{n} t} .
$$

The parameter $\rho_{s}$ can in fact be made explicit, see (36) below. The decay estimate (13) shows that the derivatives of the evolution operator can be controlled in appropriate weighted Hilbert spaces. Note however that the Lyapunov functions entering in the estimates are not the same a priori on both sides of the inequality (13). Let us emphasize, though, that we can obtain a control in all spaces $L_{\mathscr{K}_{s}}^{\infty}$ for $s$ sufficiently large (depending on the order of derivation).

\section{Analysis of the statistical error}

The asymptotic variance characterizes the statistical error. In Section 4.1, we show that the asymptotic variance is well defined for the modified Langevin dynamics. We can in fact prove a stronger result, namely that a Central Limit Theorem (CLT) holds true for ergodic averages over one trajectory. In a second step, we more carefully analyze in Section 4.2 the properties of the variance of the AR-Langevin dynamics by proving a linear response result in the limit of a vanishing lower bound on the kinetic energies. To obtain the latter results, we rely on the estimates provided by Lemma 3. 
4.1 A Central Limit theorem for ergodic averages

Let us first write the asymptotic variance in terms of the generator of the dynamics. To simplify the notation, we introduce the orthogonal projection $\Pi_{\mu}$ onto the orthogonal of the kernel of the operator $\mathscr{L}$ (with respect to the $L^{2}(\mu)$ scalar product): for any $\psi \in L^{2}(\mu)$,

$$
\Pi_{\mu} \psi:=\psi-\int_{\mathscr{E}} \psi d \mu
$$

Since $L_{\mathscr{K}_{s}}^{\infty} \subset L^{2}(\mu)$, we can define $\widetilde{L_{\mathscr{K}_{s}}^{\infty}}=\Pi_{\mu}\left(L_{\mathscr{K}_{s}}^{\infty}\right)$. The ergodicity result (9) allows us to conclude that the operator $\mathscr{L}$ is invertible on $\widetilde{L_{\mathscr{K}_{S}}^{\infty}}$ since the following operator equality holds on $\mathscr{B}\left(\widetilde{L_{\mathscr{K}_{s}}^{\infty}}\right)$, the Banach space of bounded operators on $\widetilde{L_{\mathscr{K}_{s}}^{\infty}}$ :

$$
\mathscr{L}^{-1}=\int_{0}^{+\infty} \mathrm{e}^{t \mathscr{L}} d t .
$$

This leads to the following resolvent bounds (the second part being a direct corollary of Lemma 3).

Corollary 1 Suppose that Assumption 2 holds. Then, for any $s \in \mathbb{N}^{*}$,

$$
\left\|\mathscr{L}^{-1}\right\|_{\mathscr{B}\left(\widetilde{L_{\mathscr{K}}^{\infty}}\right)} \leqslant \frac{C_{s}}{\lambda_{s}}
$$

where $\lambda_{s}, C_{s}$ are the constants introduced in Theorem 4. Suppose in addition that Assumption 5 holds, and fix $A \in \mathscr{S}$. For any $n \geqslant 1$, there exist $\widetilde{n}, s_{n} \in \mathbb{N}$ and $\lambda_{n}>0$ such that, for $s \geqslant s_{n}$ and $G_{v} \leqslant \rho_{s}$ with $\rho_{s}>0$ sufficiently small (depending on $s$ but not on $n$ ), there is $r \in \mathbb{N}$ and $C>0$ for which

$$
\forall|k| \leqslant n, \quad\left\|\partial^{k} \mathscr{L}^{-1} \Pi_{\mu} A\right\|_{L_{\mathscr{K}_{s}}^{\infty}} \leqslant \frac{C}{\lambda_{n}}\|A\|_{W_{\mathscr{K}_{r}}^{\widetilde{n}, \infty}} .
$$

This already allows us to conclude that the asymptotic variance of the time average $\widehat{A}_{t}$ defined in (1) is well defined for any observable $A \in L_{\mathscr{K}_{r}}^{\infty}$ since

$$
\begin{aligned}
\sigma_{A}^{2} & =\lim _{t \rightarrow \infty} t \mathbb{E}\left[\left(\hat{A}_{t}-\mathbb{E}_{\mu}(A)\right)^{2}\right] \\
& =\lim _{t \rightarrow \infty} 2 \int_{0}^{t} \int_{\mathscr{E}}\left(1-\frac{s}{t}\right)\left(\mathrm{e}^{\mathrm{s} \mathscr{L}} \Pi_{\mu} \mathrm{A}\right)\left(\Pi_{\mu} A\right) d \mu \\
& =2 \int_{0}^{\infty} \int_{\mathscr{E}}\left(\mathrm{e}^{s \mathscr{L}} \Pi_{\mu} A\right)\left(\Pi_{\mu} A\right) d \mu
\end{aligned}
$$

by the dominated convergence theorem. Therefore,

$$
\sigma_{A}^{2}=2 \int_{\mathscr{E}}\left(\Pi_{\mu} A\right)\left(-\mathscr{L}^{-1} \Pi_{\mu} A\right) d \mu .
$$

In fact, a Central Limit Theorem can be shown to hold for $\widehat{A}_{t}$ using standard results (see e.g. Bhattacharya (1982)). 
4.2 Perturbative study of the variance for the AR-Langevin dynamics

Our aim in this section is to better understand, from a quantitative viewpoint, the behavior of the asymptotic variance for the AR-Langevin dynamics defined in Section 2.1, at least in some limiting regime where the parameter $K_{\min }$ is small. For intermediate values, we need to rely on numerical simulations (see Section 5).

The regime where both $K_{\min }$ and $K_{\max }$ go to 0 is somewhat singular since the transition from $U(p)=0$ to $U(p)=U_{\text {std }}(p)$ becomes quite abrupt, which prevents a rigorous theoretical analysis. The regimes where either $K_{\min }$ or $K_{\max }$ go to infinity are also of dubious interest since the dynamics strongly perturbs the standard Langevin dynamics. Therefore, we restrict ourselves to the situation where $K_{\min } \rightarrow 0$ with $K_{\max }$ fixed.

In order to highlight the dependence of the AR kinetic energy function on the restraining parameters $0 \leqslant K_{\min }<K_{\max }$, we denote it by $U_{K_{\min }, K_{\max }}$ in the remainder of this section. Let us however first give a more precise definition of this function, having in mind that $K_{\max }$ is fixed while $K_{\min }$ eventually goes to 0 . We introduce to this end an interpolation function $f_{0, K_{\max }} \in C^{\infty}(\mathbb{R})$ such that

$$
0 \leqslant f_{0, K_{\max }} \leqslant 1, \quad f_{0, K_{\max }}(x)=1 \text { for } x \leqslant 0, \quad f_{0, K_{\max }}(x)=0 \text { for } x \geqslant K_{\max },
$$

and

$$
\forall n \geqslant 1, \quad f_{0, K_{\max }}^{(n)}(0)=f_{0, K_{\max }}^{(n)}\left(K_{\max }\right)=0 .
$$

We next define an interpolation function $f_{K_{\min }, K_{\max }}$ obtained from the function $f_{0, K_{\max }}$ by an appropriate shift of the lower bound and a rescaling. More precisely, $f_{K_{\min }, K_{\max }}(x)=$ $f_{0, K_{\max }}\left(\theta_{K_{\min }}(x)\right)$ with

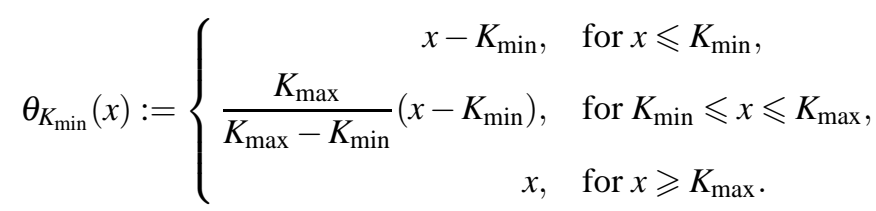

A plot of $f_{K_{\min }, K_{\max }}$ is provided in Figure 3.

Definition 1 (AR kinetic energy function) For two parameters $0 \leqslant K_{\min }<K_{\max }$, the AR kinetic energy function $U_{K_{\min }, K_{\max }}$ is defined as

$$
U_{K_{\min }, K_{\max }}(p):=\sum_{i=1}^{N} u_{K_{\min }, K_{\max }}\left(p_{i}\right)
$$

where the individual kinetic energy functions are

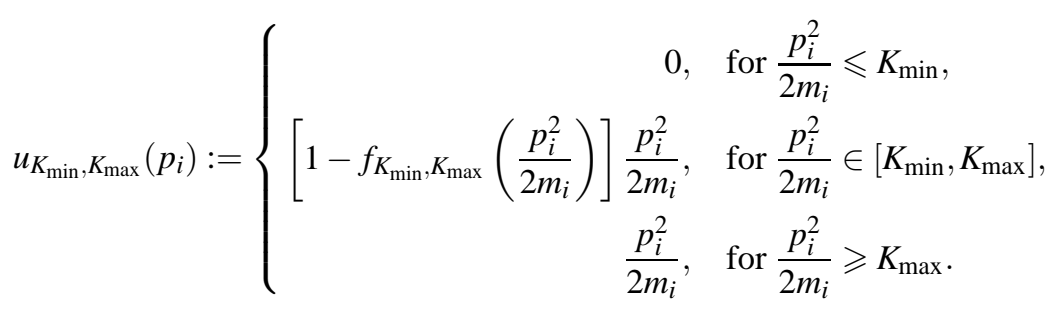




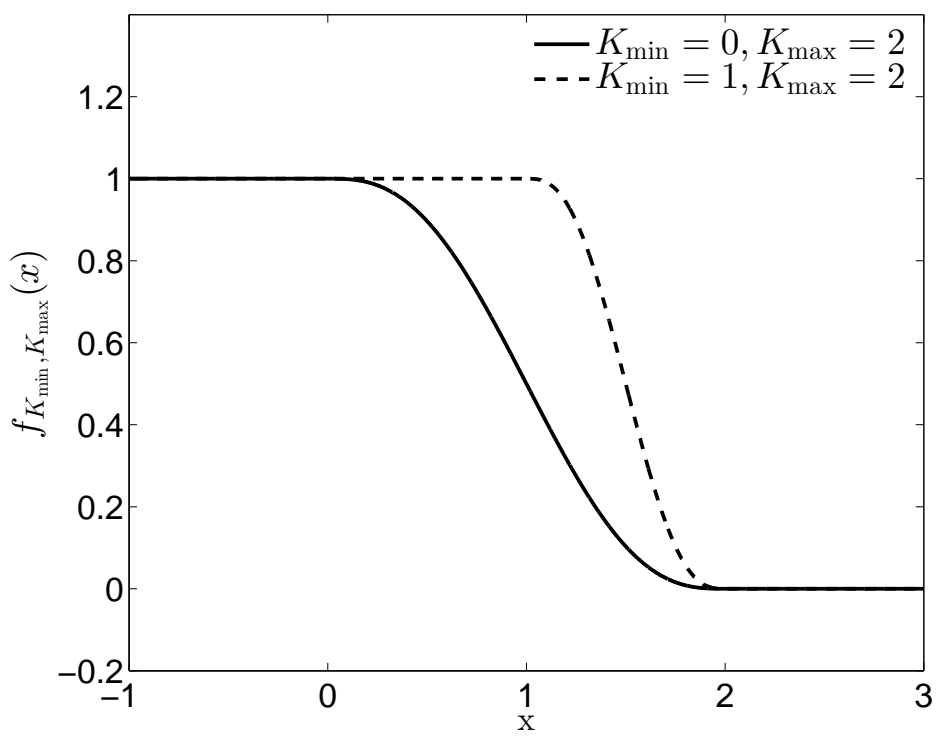

Fig. 3: Functions $f_{0, K_{\max }}$ and $f_{K_{\min }, K_{\max }}$ for $K_{\max }=2$ and $K_{\min }=1$.

Of course, $U_{K_{\min }, K_{\max }}(p)$ converges to $U_{0, K_{\max }}(p)$ as $K_{\min } \rightarrow 0$. The limiting kinetic energy function $U_{0, K_{\max }}$ corresponds to what we call the Zero- $K_{\max }$-AR-Langevin dynamics (see Figure 4 for an illustration). Let us emphasize that the limiting dynamics is not the standard Langevin dynamics, so that the expansion in powers of $K_{\min }$ of the variance we provide is with respect to the limiting variance of the dynamics corresponding to $U_{0, K_{\max }}$. To simplify the notation, we denote by $\sigma^{2}\left(K_{\min }\right)$ the variance associated with the kinetic energy $U_{K_{\min }, K_{\max }}$.

Proposition 1 There exists $K_{\max }^{*}>0$ such that, for any $0<K_{\max } \leqslant K_{\max }^{*}$, there is a constant $\mathscr{K}>0$ for which

$$
\forall 0 \leqslant K_{\min } \leqslant \frac{K_{\max }}{2}, \quad \sigma_{A}^{2}\left(K_{\min }\right)=\sigma_{A}^{2}(0)+\mathscr{K} K_{\min }+\mathrm{O}\left(K_{\text {min }}^{2}\right) .
$$

The proof can be read in Section 6.4. The assumption that $K_{\max }$ is sufficiently small ensures that Assumption 5 holds (see Section 6.4.3). The result is formally clear. The difficulty in proving it is that the kinetic energy is not a smooth function of $K_{\min }$ because the shift function is only piecewise smooth.

Remark 3 An inspection of the proof of Proposition 1 shows that the linear response result can be generalized to non-zero values of $K_{\min }$ and in fact to linear responses in the parameter $K_{\max }$ as well. For the latter case, we consider $f_{0, K_{\max }}(x)=f_{0,1}\left(x / K_{\max }\right)$. Denoting now by $\sigma^{2}\left(K_{\min }, K_{\max }\right)$ the variance associated with the kinetic energy $U_{K_{\min }, K_{\max }}$, it can be proved that, for $0<K_{\min }<K_{\max }$ not too large, there are $a, b \in \mathbb{R}$ such that, for $\delta, \eta \in \mathbb{R}$ sufficiently small,

$$
\sigma^{2}\left(K_{\min }+\delta, K_{\max }+\eta\right)=\sigma^{2}\left(K_{\min }, K_{\max }\right)+a \delta+b \eta+\mathrm{O}\left(\eta^{2}+\delta^{2}\right) .
$$




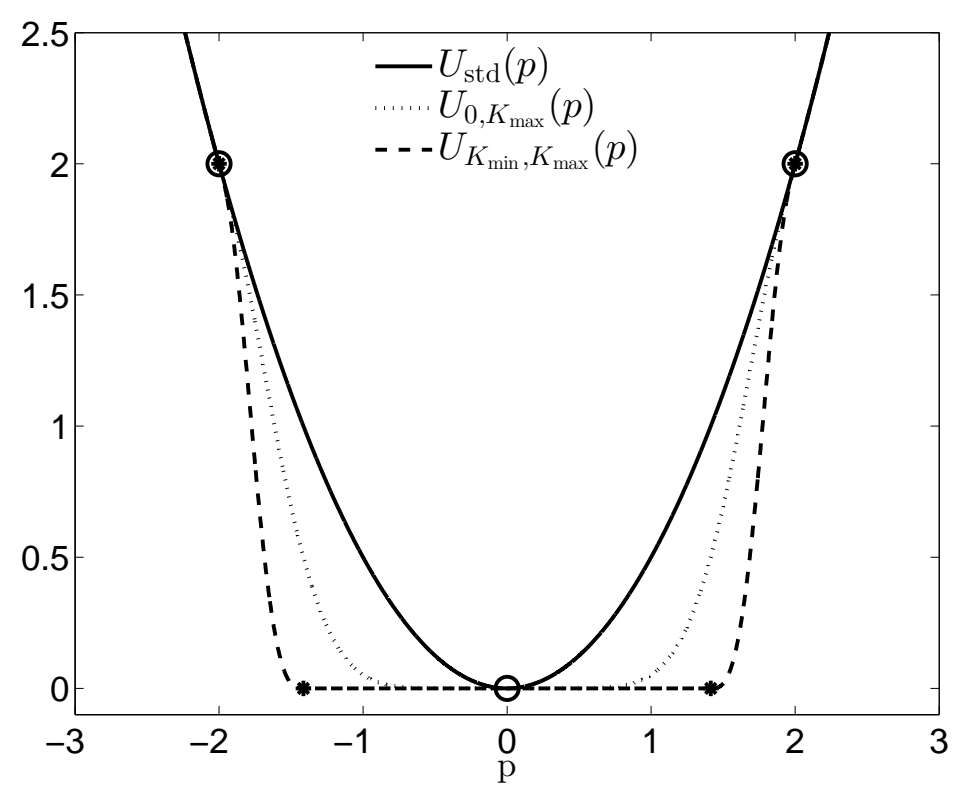

Fig. 4: Standard kinetic energy function $U_{\text {std }}$, as well as two AR kinetic energy functions $U_{K_{\min }, K_{\max }}$ with $K_{\max }=2$ and $K_{\min }=0$ or 1 .

\section{Numerical results}

The aim of this section is to quantify the evolution of the variance of AR-Langevin dynamics as the parameters of the kinetic energy function are modified. We first consider in Section 5.1 a simple system in spatial dimension 1, for which the variance can be very precisely computed using a Galerkin-type approximation. We next consider more realistic particle systems in Section 5.2, relying on molecular dynamics simulations to estimate the variance. In this section, the function $f_{0, K_{\max }}(x)$ is chosen to be of the form $f_{0,1}\left(x / K_{\max }\right)$, with $f_{0,1}$ a fifth-order spline function.

\subsection{A simple one-dimensional system}

We first consider a single particle in spatial dimension $d=1$, in the periodic domain $\mathscr{D}=2 \pi \mathbb{T}$ and at inverse temperature $\beta=1$. In this case, it is possible to directly approximate the asymptotic variance (16) using some Galerkin discretization, as in Risken (1984) or Latorre et al. (2013).

We denote by $\mathscr{L}_{K_{\min }, K_{\max }}$ the generator of the modified Langevin dynamics associated with the AR kinetic energy function $U_{K_{\min }, K_{\max }}$ defined in (19), by $\mu_{K_{\min }, K_{\max }}$ the associated canonical measure, and by $\Pi_{K_{\min }, K_{\max }}$ the projector onto functions of $L^{2}\left(\mu_{K_{\min }, K_{\max }}\right)$ with average 0 with respect to $\mu_{K_{\min }, K_{\max }}$. 
For a given observable $A$, we first approximate the solution of the following Poisson equation:

$$
-\mathscr{L}_{K_{\min }, K_{\max }} \Phi_{A}=\Pi_{K_{\min }, K_{\max }} A,
$$

and then compute the variance as given by (16):

$$
\sigma_{A}^{2}=2 \int_{\mathscr{E}} \Phi_{A} A d \mu_{K_{\min }, K_{\max }}
$$

To achieve this, we introduce the basis functions $\psi_{n k}(q, p):=G_{k}(q) H_{n}(p)$, where $G_{k}(q)=(2 \pi)^{-1 / 2} \mathrm{e}^{\mathrm{i} k q}$ (for $k \in \mathbb{Z}$ ) and $H_{n}(p)$ are the Hermite polynomials:

$$
H_{n}(p)=(-1)^{n} \mathrm{e}^{p^{2} / 2} \frac{d^{n}}{d p^{n}}\left(\mathrm{e}^{-p^{2} / 2}\right), \forall n \in \mathbb{N} .
$$

The choice of $G_{k}$ is natural in view of the spatial periodicity of the functions under consideration, while Hermite polynomials are eigenfunctions of the generator associated with the Ornstein-Uhlenbeck process on the momenta for the standard quadratic kinetic energy $p^{2} / 2$. Note however that, when the kinetic energy is modified as $U_{K_{\min }, K_{\max }}$, the Hermite polynomials are no longer orthogonal for the $L^{2}\left(\mu_{K_{\min }, K_{\max }}\right)$ scalar product.

We approximate the Poisson equation (22) on the basis

$\mathscr{V}_{N_{G}, N_{H}}=\left\{\psi_{n k}\right\}_{0 \leqslant n \leqslant N_{H},-N_{G} \leqslant k \leqslant N_{G}}$ for given integers $N_{G}, H_{H} \geqslant 1$, and we look for approximate solutions of the form $\Pi_{K_{\min }, K_{\max }} \Phi_{A}^{N_{G}, N_{H}}$ with

$$
\Phi_{A}^{N_{G}, N_{H}}=\sum_{n=-N_{H}}^{N_{H}} \sum_{k=0}^{N_{G}}\left[b_{N_{G}, N_{H}}\right]_{n k} \psi_{n k},
$$

where $b_{N_{G}, N_{H}}=\left(b_{n k}\right)_{0 \leqslant n \leqslant N_{H},-N_{G} \leqslant k \leqslant N_{G}}$ is a vector of size $\left(2 N_{G}+1\right)\left(N_{H}+1\right)$. Restricting (22) to $\mathscr{V}_{N_{G}, N_{H}}$ leads to

$$
M_{N_{G}, N_{H}} b_{N_{G}, N_{H}}=a_{N_{G}, N_{H}}
$$

where $M_{N_{G}, N_{H}}$ is a matrix of size $\left(2 N_{G}+1\right)\left(N_{H}+1\right) \times\left(2 N_{G}+1\right)\left(N_{H}+1\right)$ and $a_{N_{G}, N_{H}}$ a vector of size $\left(2 N_{G}+1\right)\left(N_{H}+1\right)$, whose entries respectively read

$$
\begin{aligned}
{\left[M_{N_{G}, N_{H}}\right]_{n k, m l} } & =\left\langle\psi_{m l},-\mathscr{L}_{K_{\min }, K_{\max }} \psi_{n k}\right\rangle_{L^{2}\left(\mu_{K_{\min }, K_{\max }}\right)}, \\
{\left[a_{N_{G}, N_{H}}\right]_{m l} } & =\left\langle\psi_{m l}, \Pi_{K_{\min }, K_{\max }} A\right\rangle_{L^{2}\left(\mu_{K_{\min }, K_{\max }}\right)} .
\end{aligned}
$$

The approximated solution $\Phi_{A}^{N_{G}, N_{H}}$ of the Poisson equation (22) can therefore be computed by solving (23). Note however that some care is needed at this stage since $\mathscr{L}_{K_{\min }, K_{\max }}$ is not invertible on $\mathscr{V}_{N_{G}, N_{H}}$, because the basis functions $\left\{\psi_{n k}\right\}_{0 \leqslant n \leqslant N_{H},-N_{G} \leqslant k \leqslant N_{G}}$ are not of integral 0 with respect to $\mu_{K_{\min }, K_{\max }}$. We correct this by performing a singular value decomposition of $M_{N_{G}, N_{H}}$, removing the component of $a_{N_{G}, N_{H}}$ associated with the singular value 0 , and computing the inverse of $M_{N_{G}, N_{H}}$ on the subspace generated by the eigenvectors associated with non-zero eigenvalues. In practice, we compute the entries of $a_{N_{G}, N_{H}}$ and $M_{N_{G}, N_{H}}$ by numerical quadrature. Since the Hermite 


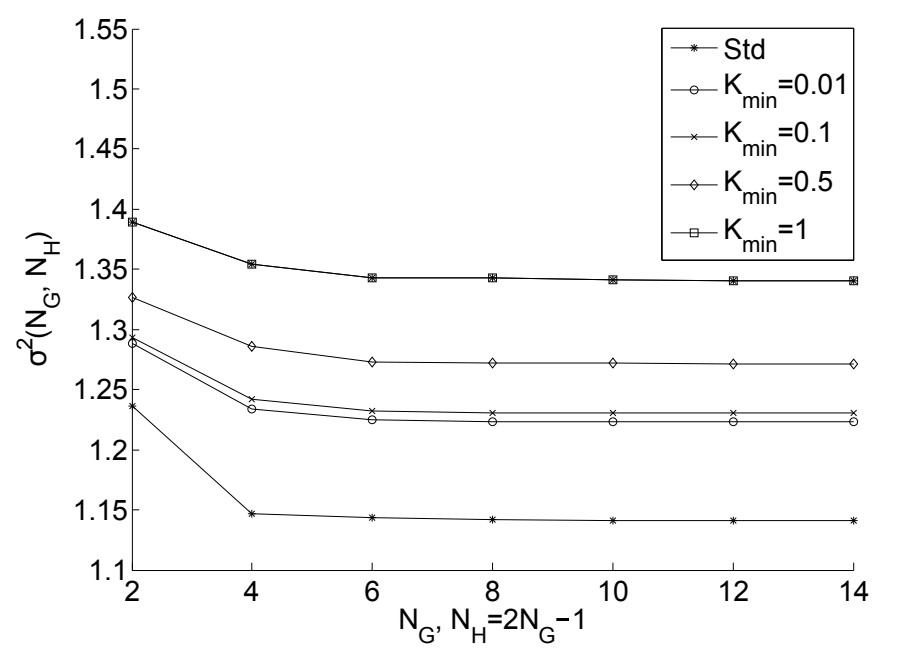

Fig. 5: Convergence of the Galerkin approximation in the basis size $N_{G}$ and $N_{H}=$ $2 N_{G}-1$ : approximation of the variance of observable $A=V$ for the standard dynamics and the AR dynamics with fixed parameter $K_{\max }=2$ and various values of $K_{\min }$.

polynomials are no longer orthogonal for the $L^{2}\left(\mu_{K_{\min }, K_{\max }}\right)$ scalar product, quadratures are required both in position and momentum variables. The variance is finally approximated as

$$
\sigma_{A}^{2}\left(N_{G}, N_{H}\right)=2 \int_{\mathscr{E}} A \Phi_{A}^{N_{G}, N_{H}} d \mu_{K_{\min }, K_{\max }}=2 b_{N_{G}, N_{H}}^{T} a_{N_{G}, N_{H}}
$$

In the simulations presented in this section, the potential is $V(q)=\cos (q)$, the observable under study is $A=V$, and we always set $N_{H}=2 N_{G}-1$. Figure 5 presents the convergence of the variance with respect to the basis size, for the standard Langevin dynamics and the AR Langevin dynamics with $K_{\max }=2$ and various values of $K_{\min }$. The results show that the choice $N_{G}=12$ is sufficient in all cases to approximate the asymptotic value. We checked in addition in one case, namely for the standard dynamics, that the values we obtain are very close to a reference value obtained with $N_{G}=30$ : the relative variation is of order $10^{-8}$ for $N_{G}=10,10^{-10}$ for $N_{G}=12$ and $10^{-11}$ for $N_{G}=14$. We therefore set $N_{G}=12$ in the remainder of this section.

The variation of the computed variance for $A=V$ is plotted in Figure 6 for various parameters $0 \leqslant K_{\min }<K_{\max }$ of the AR-Langevin dynamics. Note that, as expected, the variance increases with increasing values of $K_{\min }$ for fixed $K_{\max }$, but also with increasing values of $K_{\max }$ for fixed $K_{\min }$. We next illustrate the linear response results of Proposition 1 and Remark 3 in Figures 7 and 8: in both situations, the variance increases linearly with the parameter under consideration is varied in a sufficiently small neighborhood of its initial value. After that initial regime, nonlinear variations 


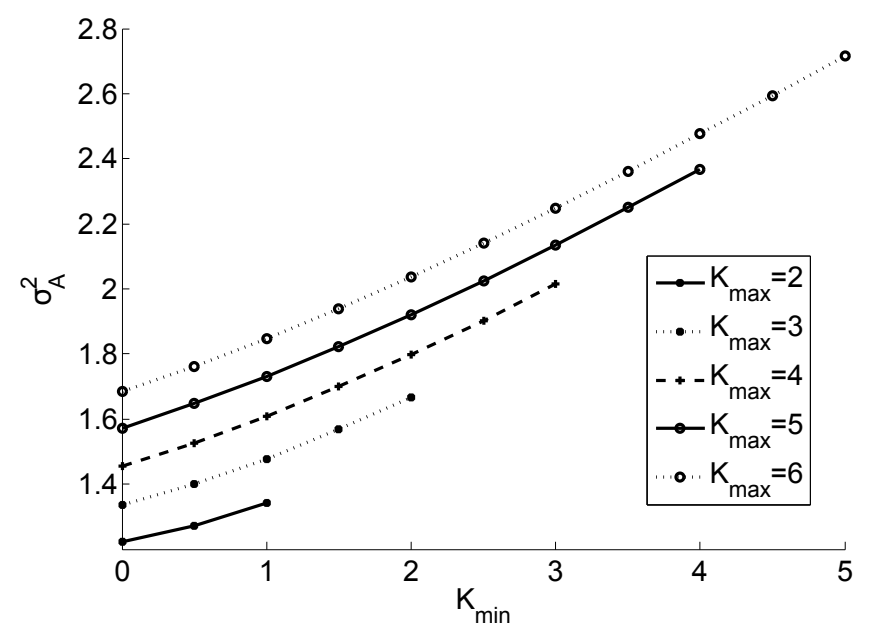

Fig. 6: Asymptotic variance of time averages for $A=V$, approximated by the Galerkin method, as a function of $K_{\min }$ and for several values of $K_{\max }$.

appear. Note also that the relative increase of the variance is more pronounced as a function of $K_{\max }$ than $K_{\min }$.

Remark 4 In practice, the idea usually is to set the lower bound $K_{\min }$ sufficiently large when performing Monte Carlo simulations, in order to decrease as much as possible the computational cost. The gap $K_{\max }-K_{\min }$ should however not be too small in order to have a sufficiently smooth transition from a vanishing kinetic energy to a quadratic one. This requires therefore $K_{\max }$ to be quite large if $K_{\min }$ is large. The results presented in Figure 8 suggest that this may not be the optimal choice, unless the algorithmic speed-up is quite large.

\subsection{A more realistic system}

In order to study the variation of the variance as a function of $K_{\min }$ and $K_{\max }$ in systems of higher dimensions, we resort to Monte Carlo simulations. This requires discretizing the AR-Langevin dynamics (5), and we resort to a scheme of weak order 2, obtained by a splitting strategy where the generator of the modified Langevin dynamics (6) is decomposed into three parts:

$$
A:=\nabla U(p) \cdot \nabla_{q}, \quad B:=-\nabla V(q) \cdot \nabla_{p}, \quad C:=-\nabla U(p) \cdot \nabla_{p}+\frac{1}{\beta} \Delta_{p} .
$$

The transition kernel obtained by a Strang splitting reads $P_{\Delta t}=\mathrm{e}^{\gamma \Delta t C / 2} \mathrm{e}^{\Delta t B / 2} \mathrm{e}^{\Delta t A} \mathrm{e}^{\Delta t B / 2} \mathrm{e}^{\gamma \Delta t C / 2}$. Contrarily to the standard kinetic energy functions, the elementary evolution associated with $C$ cannot be integrated analytically. To preserve the order of the scheme, we 


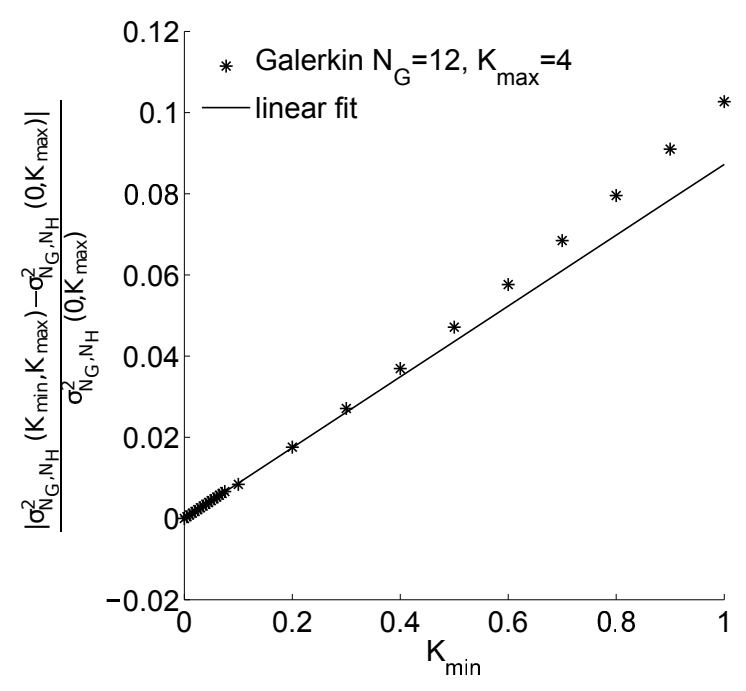

Fig. 7: Relative difference between the variance $\sigma_{N_{G}, N_{H}}^{2}\left(K_{\min }, K_{\max }\right)$ and its initial value computed for reference parameters. (Left) Fixed upper bound $K_{\max }=4$, and reference value $K_{\min }=0$.

approximate $\mathrm{e}^{\gamma \Delta t / 2 C}$ by a midpoint rule, encoded by a transition kernel $P_{\Delta t}^{\gamma, C}$ satisfying $P_{\Delta t}^{\gamma, C} \varphi=\mathrm{e}^{\gamma \Delta t C} \varphi+\mathrm{O}\left(\Delta t^{3}\right)$ for smooth test functions $\varphi$. This gives the following discretization scheme:

$$
\left\{\begin{aligned}
p^{n+1 / 4} & =p^{n}-\gamma \nabla U\left(\frac{p^{n+1 / 4}+p^{n}}{2}\right) \frac{\Delta t}{2}+\sqrt{\frac{\gamma \Delta t}{\beta}} G^{n}, \\
p^{n+1 / 2} & =p^{n+1 / 4}-\nabla V\left(q^{n}\right) \frac{\Delta t}{2} \\
q^{n+1} & =q^{n}+\nabla U\left(p^{n+1 / 2}\right) \Delta t \\
p^{n+3 / 4} & =p^{n+1 / 2}-\nabla V\left(q^{n+1}\right) \frac{\Delta t}{2} \\
p^{n+1} & =p^{n+3 / 4}-\gamma \nabla U\left(\frac{p^{n+1}+p^{n+3 / 4}}{2}\right) \frac{\Delta t}{2}+\sqrt{\frac{\gamma \Delta t}{\beta}} G^{n+1 / 2},
\end{aligned}\right.
$$

where $G^{n}, G^{n+1 / 2}$ are i.i.d. standard $d$-dimensional Gaussian random variables. The first and the last line are obtained by implicit schemes, solved in practice by a fixed point strategy (the termination criterion being that the distance between successive iterates is smaller than $10^{-10}$, and the initial iterate being obtained by a Euler-Maruyama step). By following the same approach as in Leimkuhler et al. (2015), it can indeed be proved that this scheme is of weak order 2; see Stoltz and Trstanova (In preparation) for further precisions. 


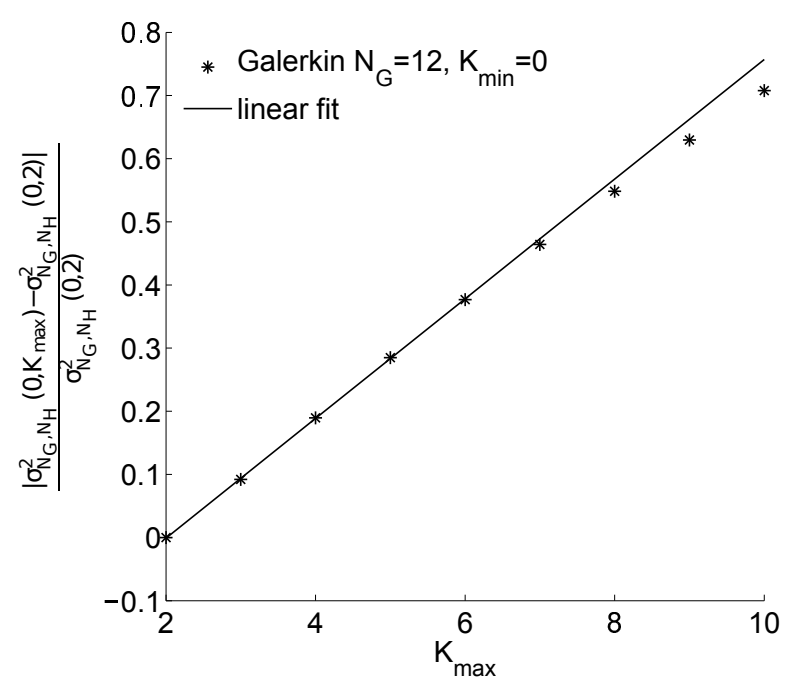

Fig. 8: Same as Figure 7. Fixed lower bound $K_{\min }=0$, and reference value $K_{\max }=2$.

The ergodicity of some second-order schemes was proved for the standard Langevin dynamics in Leimkuhler et al. (2015). Since the AR-Langevin dynamics can be seen as a perturbation of the standard Langevin dynamics, it can be proved by combining the proofs from Leimkuhler et al. (2015) and the proof of Theorem 4 that, when $0 \leqslant K_{\min }<K_{\max }$ are sufficiently small, the corresponding discretization of the ARLangevin dynamics remains ergodic (see Stoltz and Trstanova (In preparation)). The corresponding invariant measure is denoted by $\mu_{K_{\min }, \Delta t}$. It also follows by the results of Leimkuhler et al. (2015) that the error on averages of smooth observables $\varphi \in \mathscr{S}$ with respect to $\mu_{K_{\min }, \Delta t}$ is of order 2 , i.e. there exists $a \in \mathbb{R}$ such that

$$
\int_{\mathscr{E}} \varphi d \mu_{K_{\min }, \Delta t}=\int_{\mathscr{E}} \varphi d \mu_{K_{\min }}+a \Delta t^{2}+\mathrm{O}\left(\Delta t^{3}\right) .
$$

As already mentioned in Remark 4, the reduction of the gap between the parameters $K_{\min }$ and $K_{\max }$ reduces the smoothness of the transition between the restrained dynamics and the full dynamics. This raises issues in the stability of the scheme, which can be partly cured by resorting to a Metropolis-Hastings correction (Metropolis et al. (1953); Hastings (1970) and Stoltz and Trstanova (In preparation)).

The system we consider is composed of $N=49$ particles in dimension 2, so that $d=98$ and $\mathscr{D}=(L \mathbb{T})^{2 N}$. The masses are set to 1 for all particles. Among these particles, two particles (numbered 1 and 2 in the following) are designated to form a dimer while the others are solvent particles. All particles, except the two particles forming the dimer, interact through the purely repulsive WCA pair potential, which is a truncated Lennard-Jones potential Straub et al. (1988):

$$
V_{\mathrm{WCA}}(r)=\left\{\begin{array}{cc}
4 \varepsilon\left[\left(\frac{\sigma}{r}\right)^{12}-\left(\frac{\sigma}{r}\right)^{6}\right]+\varepsilon & \text { if } r \leqslant r_{0}, \\
0 & \text { if } r>r_{0},
\end{array}\right.
$$


where $r$ denotes the distance between two particles, $\varepsilon$ and $\sigma$ are two positive parameters and $r_{0}=2^{1 / 6} \sigma$. The interaction potential between the two particles of the dimer is a double-well potential

$$
V_{\mathrm{D}}(r)=h\left[1-\frac{\left(r-r_{0}-w\right)^{2}}{w^{2}}\right]^{2},
$$

where $h$ and $w$ are two positive parameters. The potential $V_{\mathrm{D}}$ has two energy minima. The first one, at $r=r_{0}$, corresponds to the compact state. The second one, at $r=$ $r_{0}+2 w$, corresponds to the stretched state. The total energy of the system is therefore, for $q \in(L \mathbb{T})^{d N}$ with $d=2$,

$$
V(q)=V_{\mathrm{D}}\left(\left|q_{1}-q_{2}\right|\right)+V_{\mathrm{SS}}\left(q_{3}, \ldots, q_{N}\right)+V_{\mathrm{DS}}(q),
$$

where the solvent-solvent and dimer-solvent potential energies respectively read

$$
V_{\mathrm{SS}}\left(q_{3}, \ldots, q_{N}\right)=\sum_{3 \leqslant i<j \leqslant N} V_{\mathrm{WCA}}\left(\left|q_{i}-q_{j}\right|\right), \quad V_{\mathrm{DS}}(q)=\sum_{i=1,23 \leqslant j \leqslant N} \sum_{\mathrm{WCA}}\left(\left|q_{i}-q_{j}\right|\right) .
$$

We choose $\beta=1, \varepsilon_{L J}=1, \sigma_{L J}=1, h=1, w=1$, and set the particle density $\rho=$ $N / L^{2}$ to 0.56 in the numerical results presented in this section, sufficiently high to ensure that the solvent markedly modifies the distribution of configurations of the dimer compared to the gas phase.

The source of metastability in the system is the double-well potential on the dimer. In such a system, it makes sense to restrain only solvent particles (since they account for most of the computational cost), and keep the standard kinetic energy for the particles forming the dimer (since the observable depends on their positions). As noted in Remark 1, the method allows us to choose different individual kinetic energies for different particles. Since the solvent interacts with the dimer, we study how the variance of time averages of observables related to the configuration of the dimer, such as the dimer potential energy $A=V_{\mathrm{D}}$, depend on the restraining parameters chosen for the solvent particles. We also estimate the variance of time averages based on observables depending only on the solvent degrees of freedom, such as the solvent-solvent potential energy $A=V_{\mathrm{SS}}$.

The asymptotic variance of time averages for a given observable $A$ is estimated by approximating the integrated auto-correlation function

$$
\sigma_{A}^{2}=2 \int_{0}^{\infty} \mathbb{E}_{\mu_{K_{\min }, K_{\max }}}\left[\left(\Pi_{\mu} A\right)\left(q_{0}, p_{0}\right)\left(\Pi_{\mu} A\right)\left(q_{t}, p_{t}\right)\right] d t,
$$

where the expectation is with respect to initial conditions $\left(q_{0}, p_{0}\right) \sim \mu_{K_{\min }, K_{\max }}$ and all realizations of the AR Langevin dynamics. This is done by first truncating the upper bound in the integral by a sufficiently large time $T_{\text {corr }}$, and using a trapezoidal rule:

$$
\sigma_{A}^{2} \approx \sigma_{A, M, \Delta t}^{2}:=\Delta t\left(\frac{\widetilde{C}_{0}^{M}}{2}+\sum_{j=1}^{I_{\text {corr }}} \widetilde{C}_{j}^{M}\right)
$$

where $I_{\text {corr }}=\left\lfloor\frac{T_{\text {corr }}}{\Delta t}\right\rfloor$, and the empirical averages over $M$ realizations of trajectories of $I_{\text {corr }}$ steps are defined as

$$
\widetilde{C}_{j}^{M}:=C_{j}^{M}-\widehat{A}_{j}^{M} \widehat{A}_{0}^{M}, \quad j \in\left\{1, \ldots, I_{\text {corr }}\right\},
$$




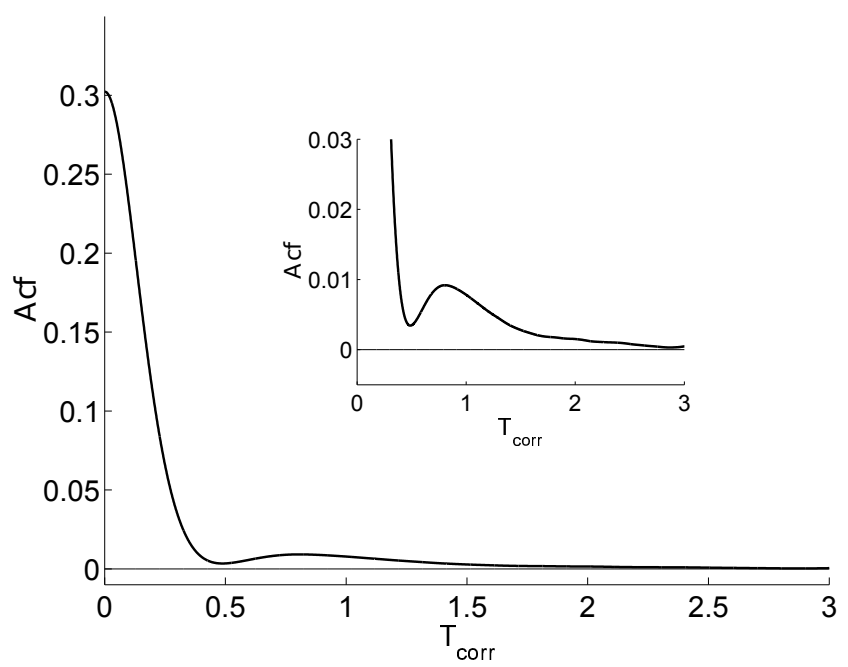

Fig. 9: Auto-correlation function $\mathbb{E}_{\mu}\left[\left(\Pi_{\mu} A\right)\left(p_{0}, q_{0}\right)\left(\Pi_{\mu} A\right)\left(p_{t}, q_{t}\right)\right]$ for $A=V_{D}$ as a function of time.

with

$$
C_{j}^{M}:=\frac{1}{M} \sum_{m=1}^{M} A\left(q_{j}^{m}, p_{j}^{m}\right) A\left(q_{0}^{m}, p_{0}^{m}\right), \quad \widehat{A}_{j}^{M}:=\frac{1}{M} \sum_{m=1}^{M} A\left(q_{j}^{m}, p_{j}^{m}\right)
$$

The initial condition $\left(q_{0}^{m+1}, p_{0}^{m+1}\right)$ for the $m+1$ th trajectory is obtained from the last configuration of the $m$ th configuration, namely $\left(q_{I_{\text {corr }}^{m+1}}^{m}, p_{I_{\text {corr }}}^{m+1}\right)$. Figure 9 presents the auto-correlation function obtained for $A=V_{\mathrm{D}}$. The results show that the choice $T_{\text {corr }}=3$ is reasonable.

The results of Leimkuhler et al. (2015); Stoltz and Trstanova (In preparation) show that the errors on the approximation of the variance should be of order $\Delta t^{2}$ when $T_{\text {corr }} \rightarrow+\infty$. This is illustrated in Figures 10,11, 12 and 13, which present the convergence of $\sigma_{A, M, \Delta t}^{2}$ as a function of $\Delta t$ for $M=3 \times 10^{6}$. It is possible to extrapolate the value of the variance at $\Delta t=0$ by fitting $\sigma_{A, M, \Delta t}^{2}$ as $a_{0}+a_{1} \Delta t^{2}$. Note that the errors on the variance are bigger in the case $K_{\min }=2.7$, which is expected due to the smaller gap between the parameters $K_{\max }, K_{\min }$. In the sequel, all the reported approximations of the variance are obtained by computing $\sigma_{A, M, \Delta t}^{2}$ for 6 values of the time step $\Delta t$, and extrapolating to the limit $\Delta t \rightarrow 0$ as in Figures 10,11, 12 and 13. More precisely, the time steps are chosen as $\Delta t_{0, k}=k \times 10^{-3}$ for $k=1, \ldots, 6$ when $K_{\min }=0$, and $\Delta t_{K_{\min }^{*}, k}=k \times 10^{-4}$ for $K_{\min }^{*}=2.7$. For intermediate values of $K_{\min }$, the time steps $\Delta t_{K_{\min }, k}$ are obtained by a linear interpolation between $\Delta t_{0, k}$ and $\Delta t_{K_{\min }^{*}, k}$.

The variations as a function of $K_{\min }$ of the approximations of the variances $\sigma_{A}^{2}\left(K_{\min }\right)$ for the solvent-solvent potential energy $V_{\mathrm{SS}}$ and the dimer potential energy $V_{\mathrm{D}}$ are reported in Figures 14 and 15. Surprisingly, even though the solvent particles are restrained, the variance of the solvent-solvent potential decreases linearly for moder- 


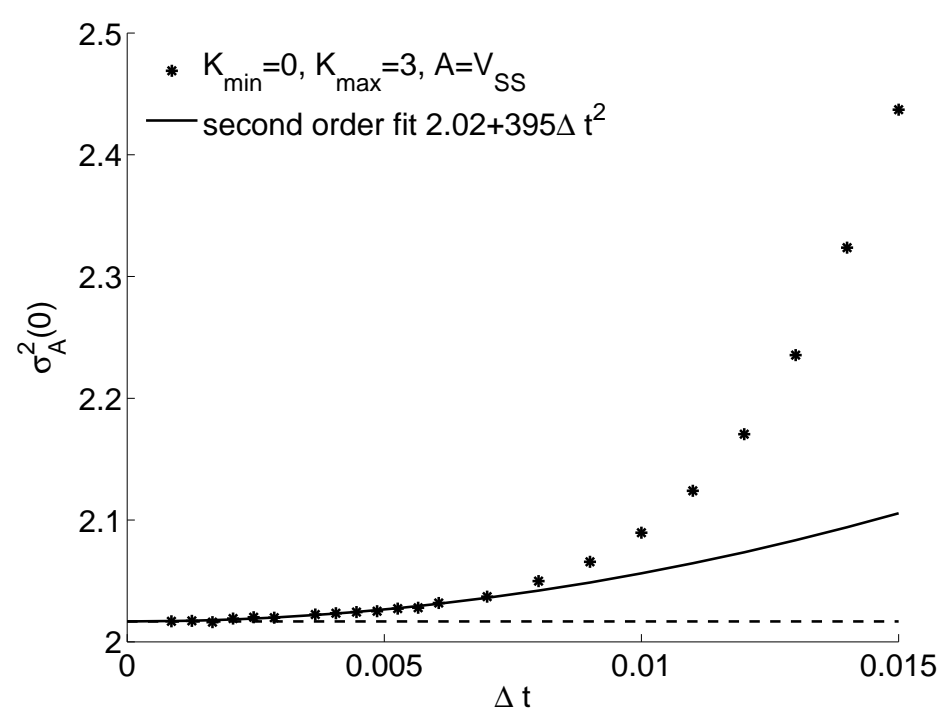

Fig. 10: Estimated variance $\sigma_{A, M, \Delta t}^{2}$ for $A=V_{\mathrm{SS}}$, as a function of the step size $\Delta t$, for $K_{\min }=0$ and $K_{\max }=3$.

ately small values of $K_{\min }$; whereas, as expected, the variance of the dimer potential, which is only implicitly influenced by the restraining parameters, increases linearly for these values of $K_{\min }$. In order to more easily compare the impacts of the restraining procedure, we plot in Figure 16 the relative differences of the variance $\sigma^{2}\left(K_{\min }\right)$ and the variance of Zero- $K_{\max }$-AR dynamics $\sigma_{A}^{2}(0)$ as a function of $K_{\min }$. For the two observables under consideration, the impact of an increase of the parameter $K_{\min }$ on the variance associated with the dimer potential is much weaker than on the variance related to the solvent potential. We also provide in Figure 17 the percentage of restrained particles, which directly depends on the restraining parameter $K_{\min }$ and dictates the algorithmic speed-up. This supports the idea that the use of the AR-Langevin method for heterogeneous systems can be beneficial when the AR parameters are set to non-zero values for the part of the system which is not directly of interest (e.g. the solvent), while the standard kinetic energy should be kept for the degrees of freedom that are directly involved in the observable (e.g. the dimer). 


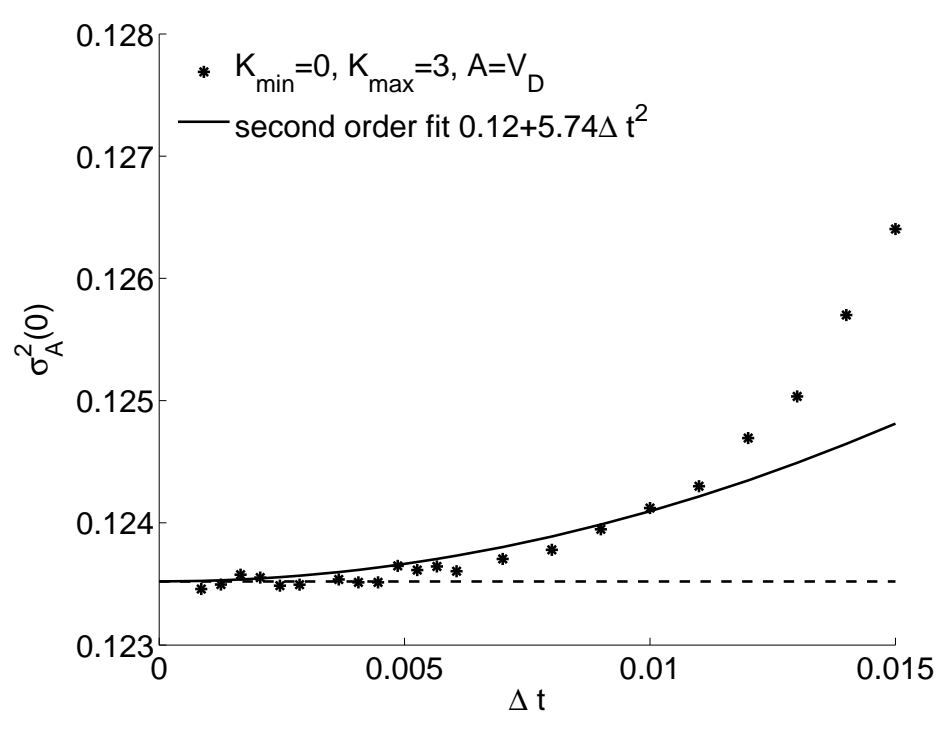

Fig. 11: Estimated variance $\sigma_{A, M, \Delta t}^{2}$ for $A=V_{\mathrm{D}}$, as a function of the step size $\Delta t$, for $K_{\min }=0$ and $K_{\max }=3$.

\section{Proofs of the results}

\subsection{Proof of Lemma 2}

The modified Langevin equation can be written as a perturbation of the Langevin equation, namely

$$
\left\{\begin{array}{l}
d q_{t}=\left(M^{-1} p_{t}-\mathscr{Z}\left(p_{t}\right)\right) d t \\
d p_{t}=-\nabla V\left(q_{t}\right) d t-\gamma\left(M^{-1} p_{t}-\mathscr{Z}\left(p_{t}\right)\right) d t+\sqrt{\frac{2 \gamma}{\beta}} d W_{t},
\end{array}\right.
$$

where $\mathscr{Z}(p):=\nabla U_{\text {std }}(p)-\nabla U(p)=M^{-1} p-\nabla U(p)$ is uniformly bounded as $|\mathscr{Z}(p)| \leqslant$ $G_{\text {std }}$ in view of Assumption 2. By a direct integration in time of the momenta dynamics,

$$
p_{t}=\mathrm{e}^{-\gamma t} p_{0}+\mathscr{F}_{t}+\mathscr{G}_{t}, \quad \mathscr{F}_{t}=\int_{0}^{t}\left(-\nabla V\left(q_{s}\right)+\gamma \mathscr{Z}\left(p_{s}\right)\right) \mathrm{e}^{-\gamma(t-s)} d s,
$$

where

$$
\mathscr{G}_{t}=\sqrt{\frac{2 \gamma}{\beta}} \int_{0}^{t} \mathrm{e}^{-\gamma(t-s)} d W_{s}
$$

is a Gaussian random variable with mean zero and covariance $\left(1-\mathrm{e}^{-2 \gamma t}\right) \beta^{-1}$. Note also that $\mathscr{F}_{t}$ is uniformly bounded; more precisely, $\left|\mathscr{F}_{t}\right| \leqslant\|\nabla V\|_{L^{\infty}} / \gamma+G_{\text {std }}$. 


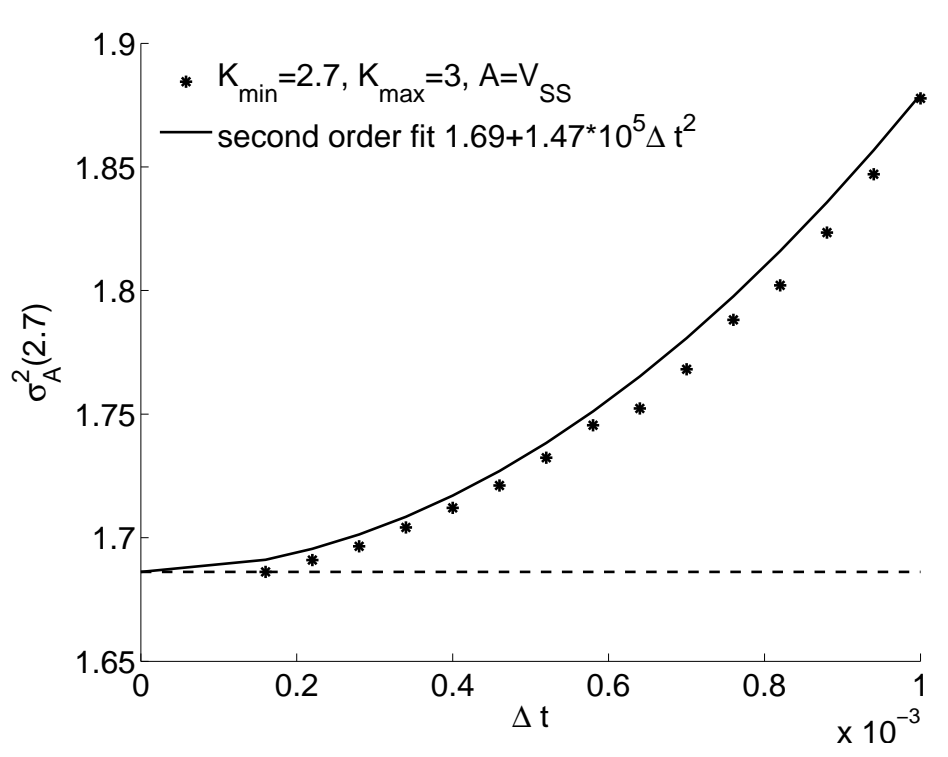

Fig. 12: Same as Figure 10, except that $K_{\min }=2.7$.

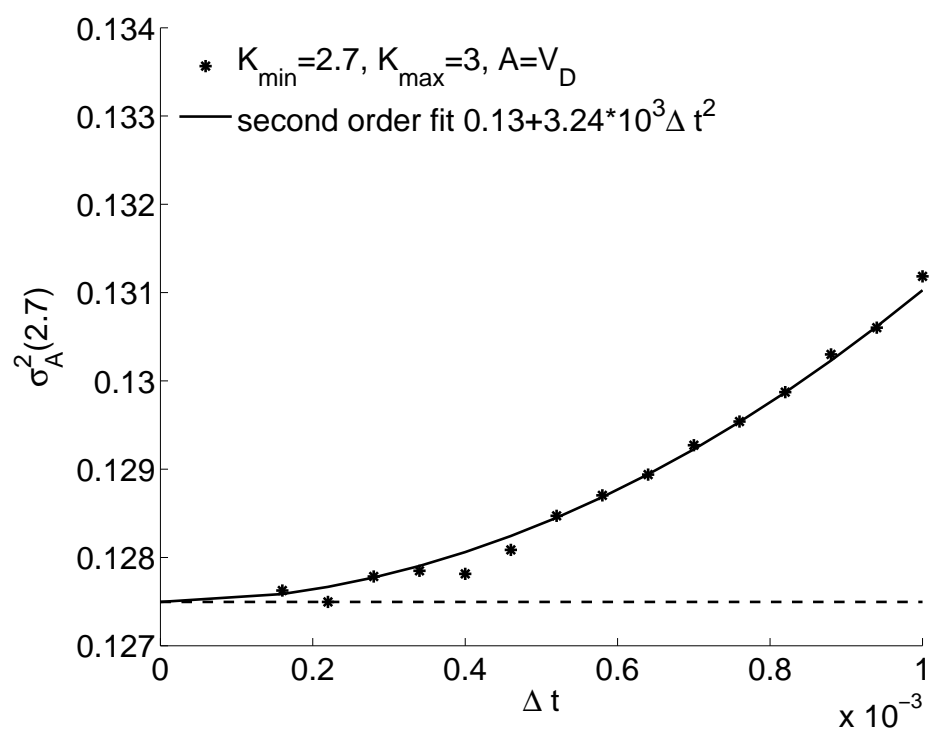

Fig. 13: Same as Figure 11, except that $K_{\min }=2.7$.

Let us first consider the case $s=1$. We introduce $\alpha_{t}:=\mathrm{e}^{-\gamma t}<1$ for a given time $t>0$. With this notation,

$$
\begin{aligned}
\left|p_{t}\right|^{2}=\left|\alpha_{t} p_{0}+\mathscr{F}_{t}+\mathscr{G}_{t}\right|^{2} & =\alpha_{t}^{2}\left|p_{t}\right|^{2}+2 \alpha_{t} p_{t}^{T}\left(\mathscr{F}_{t}+\mathscr{G}_{t}\right)+\left|\mathscr{F}_{t}\right|^{2}+2 \mathscr{F}_{t} \mathscr{G}_{t}+\left|\mathscr{G}_{t}\right|^{2} \\
& \leqslant \alpha_{t}^{2}(1+\varepsilon)\left|p_{t}\right|^{2}+\left(2+\frac{1}{4 \varepsilon}\right) \mathscr{F}_{t}^{2}+\left|\mathscr{G}_{t}\right|^{2}+2 \alpha_{t} p_{t}^{T} \mathscr{G}_{t},
\end{aligned}
$$




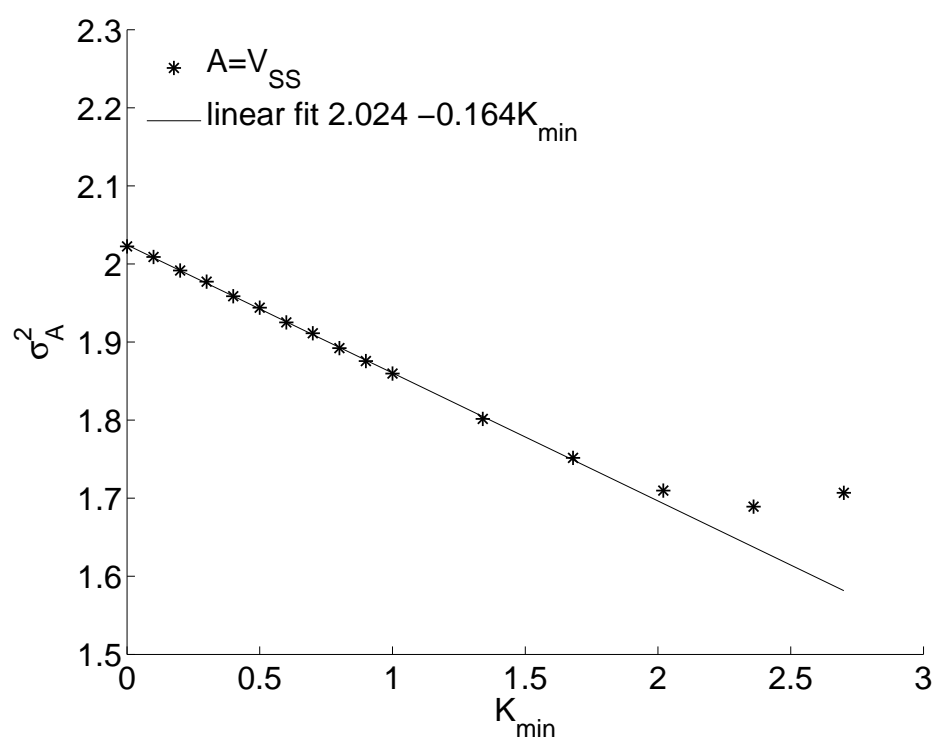

Fig. 14: Estimated variance $\sigma_{A}^{2}$ for $A=V_{\mathrm{SS}}$ as a function of $K_{\min } \in[0,2.7]$ for $K_{\max }=$ 3.

where we used Young's inequality to obtain the last line, with a constant $\varepsilon>0$ sufficiently small so that $\alpha_{t}^{2}(1+\varepsilon)<1$. We next take the expectation of the previous inequality, conditionally to the filtration of events up to time $t$. Since $\mathbb{E}\left[p_{t}^{T} \mathscr{G}_{t} \mid \mathscr{F}_{t}\right]=0$, it follows

$$
\mathbb{E}\left[\mathscr{K}_{1}\left(q_{t}, p_{t}\right) \mid \mathscr{F}_{t}\right] \leqslant \alpha^{2}(1+\varepsilon) \mathscr{K}_{1}\left(q_{t}, p_{t}\right)+R
$$

for some constant $R>0$. This shows the Lyapunov condition for $n=1$. The higher order conditions $(n>1)$ can be proved as in (Joubaud et al. 2015, Section 5.1.5), by noting that $\left|p_{t}\right|^{2 s}$ is equal to $\alpha_{t}^{2 s}\left|p_{0}\right|^{2 s}$ plus some lower order polynomial in $p_{0}$.

\subsection{Proof of Lemma 1}

The main idea is, as in (Joubaud et al. 2015, Section 5.1.5), to compare the modified Langevin dynamics to the standard Langevin dynamics with zero forces, for which a minorizing measure $v_{p^{*}, t}$ can be explicitly constructed. From the rewriting (25), we deduce, in view of the momenta evolution (26),

$$
q_{t}=q_{0}+\int_{0}^{t}\left(p_{s}-\mathscr{Z}\left(p_{s}\right)\right) d s=q_{0}+\int_{0}^{t} \mathrm{e}^{-\gamma s} p_{0} d s+\widetilde{\mathscr{G}}_{t}+\widetilde{\mathscr{F}}
$$

where periodic boundary conditions are considered, and

$$
\widetilde{\mathscr{F}_{t}}:=\int_{0}^{t} \mathscr{F}_{s} d s-\int_{0}^{t} \mathscr{Z}\left(p_{s}\right) d s, \quad \widetilde{\mathscr{G}}_{t}=\int_{0}^{t} \mathscr{G}_{s} d s .
$$




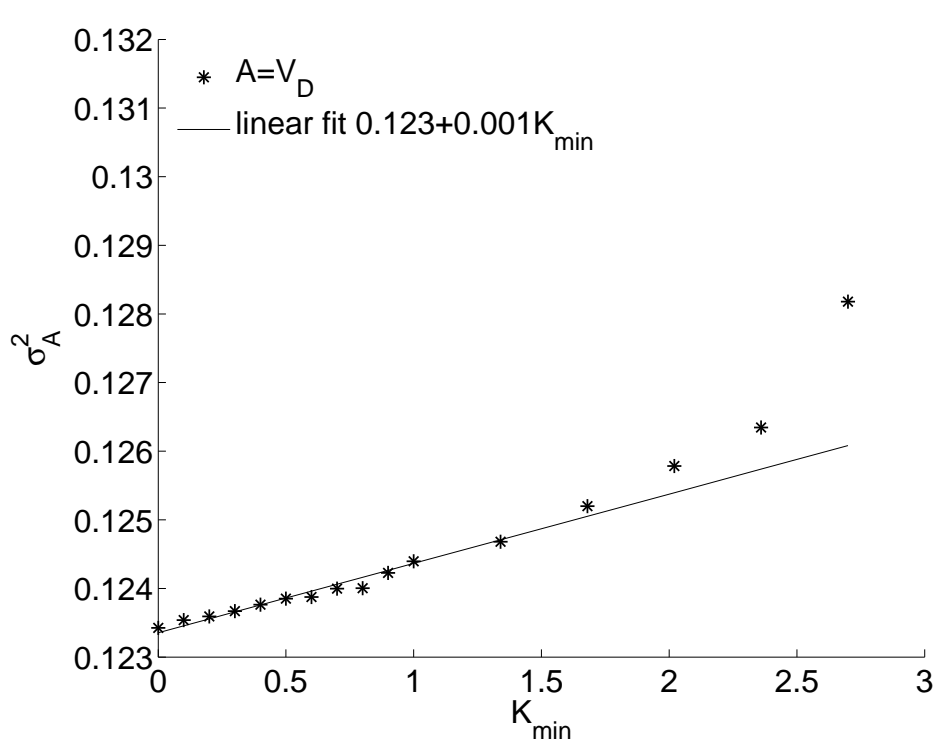

Fig. 15: Estimated variance $\sigma_{A}^{2}$ for $A=V_{\mathrm{D}}$ as a function of $K_{\min } \in[0,2.7]$ for $K_{\max }=$ 3.

Note that $\widetilde{\mathscr{F}} t$ is bounded as

$$
\left|\widetilde{\mathscr{F}_{t}}\right| \leqslant\left(\frac{\|\nabla V\|_{L^{\infty}}}{\gamma}+2 G_{\text {std }}\right) t
$$

whereas $\widetilde{\mathscr{G}}_{t}$ is a Gaussian random variable, which is correlated to $\mathscr{G}_{t}$. A simple computation shows that

$$
\mathscr{V}:=\mathbb{E}\left[\left(\widetilde{\mathscr{G}}_{t}, \mathscr{G}_{t}\right)^{T}\left(\widetilde{\mathscr{G}}_{t}, \mathscr{G}_{t}\right)\right]=\left(\begin{array}{cc}
\mathscr{V}_{q q} & \mathscr{V}_{q p} \\
\mathscr{V}_{p q} & \mathscr{V}_{p p}
\end{array}\right), \quad\left\{\begin{array}{l}
\mathscr{V}_{q q}=\frac{1}{\beta \gamma}\left(2 t-\frac{1}{\gamma}\left(3-4 \alpha_{t}+\alpha_{t}^{2}\right)\right) \\
\mathscr{V}_{q p}=\frac{1}{\beta \gamma}\left(1-\alpha_{t}\right)^{2}, \\
\mathscr{V}_{p p}=\frac{1}{\beta}\left(1-\alpha_{t}^{2}\right),
\end{array}\right.
$$

where $\alpha_{t}=\mathrm{e}^{-\gamma t}$ is the same constant as in Section 6.1. Therefore, for a given measurable set $B \in \mathscr{B}(\mathscr{E})$,

$$
\mathbb{P}\left(\left(q_{t}, p_{t}\right) \in B|| p_{0} \mid \leqslant p_{*}\right) \geqslant \mathbb{P}\left(\left(\widetilde{\mathscr{G}}_{t}, \mathscr{G}_{t}\right) \in B-\left(\mathscr{Q}_{t}, \mathscr{P}_{t}\right)|| p_{0} \mid \leqslant p_{*}\right),
$$

where

$$
\mathscr{Q}_{t}:=q_{0}+\frac{1-\alpha_{t}}{\gamma} p_{0}+\widetilde{\mathscr{F}_{t}}, \quad \mathscr{P}_{t}:=\alpha_{t} p_{0}+\mathscr{F}_{t},
$$

are both bounded by some constant $R>0$ (depending on $p^{*}$ and $t$ ) when $\left|p_{0}\right| \leqslant p^{*}$. Note that there is an inequality in (27) since we neglect in fact the periodic images of 


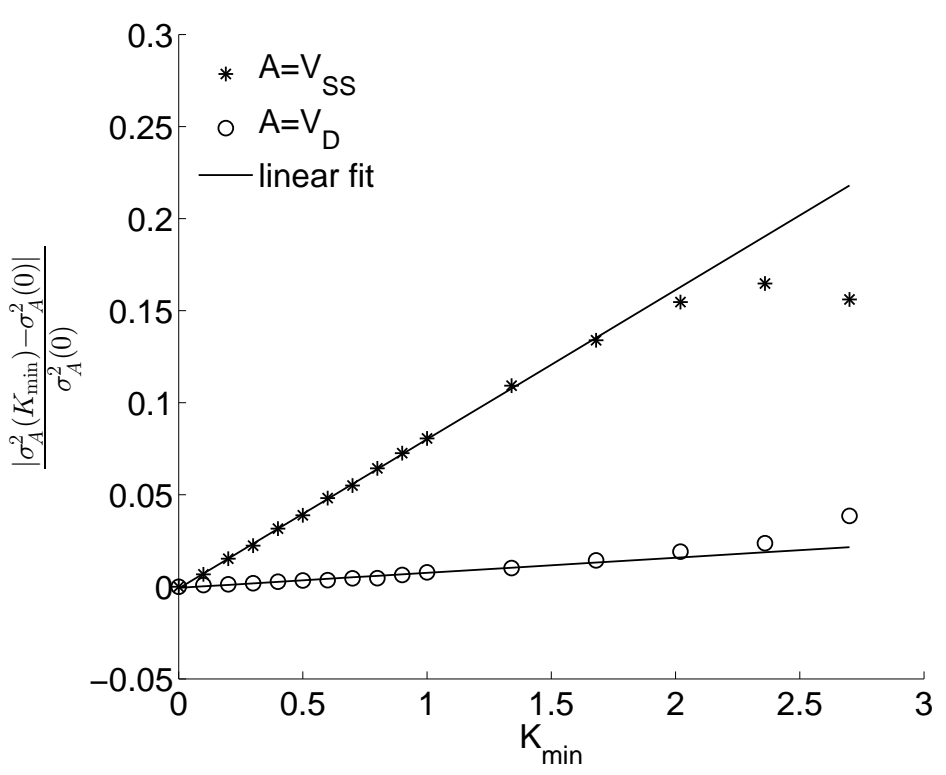

Fig. 16: Relative variation in the estimated variances $\sigma_{A}^{2}\left(K_{\min }\right)$ with respect to the reference variances $\sigma_{A}^{2}(0)$.

$q_{t}$ when writing it as $\mathscr{Q}_{t}+\widetilde{\mathscr{G}}_{t}$, the latter two quantities being interpreted as elements of $\mathbb{R}^{d}$. Since the matrix $\mathscr{V}$ is definite positive, we can finally consider the following minorizing measure:

$$
v_{p^{*}, t}(B):=Z_{R}^{-1} \inf _{|\mathscr{Q}|,|\mathscr{P}| \leqslant R} \int_{B-(\mathscr{Q}, \mathscr{P})} \exp \left(-\frac{x^{T} \mathscr{V}^{-1} x}{2}\right) d x
$$

where $Z_{R}>0$ is a normalization constant. The proof is concluded by defining $\kappa=$ $(2 \pi)^{-d} \operatorname{det}(\mathscr{V})^{-1 / 2} Z_{R}$.

\subsection{Proof of Lemma 3}

\subsubsection{General structure of the proof}

The proof follows the strategy of (Kopec 2013, Proposition A.1). We recall in this section the general outline of this proof, and highlight the required extensions. The proofs of these extensions are then provided in Section 6.3.2. Without restriction of generality, and in order to simplify the notation, we assume that $A=\Pi_{\mu} A$. We introduce weight functions

$$
\pi_{s}(p):=\frac{1}{\mathscr{K}_{s}(p)},
$$

where the Lyapunov functions $\mathscr{K}_{s}$ are defined in (8). We also define

$$
u(t, q, p)=\left(\mathrm{e}^{t \mathscr{L}} A\right)(q, p)=\mathbb{E}\left[A\left(q_{t}, p_{t}\right) \mid\left(q_{0}, p_{0}\right)=(q, p)\right] .
$$




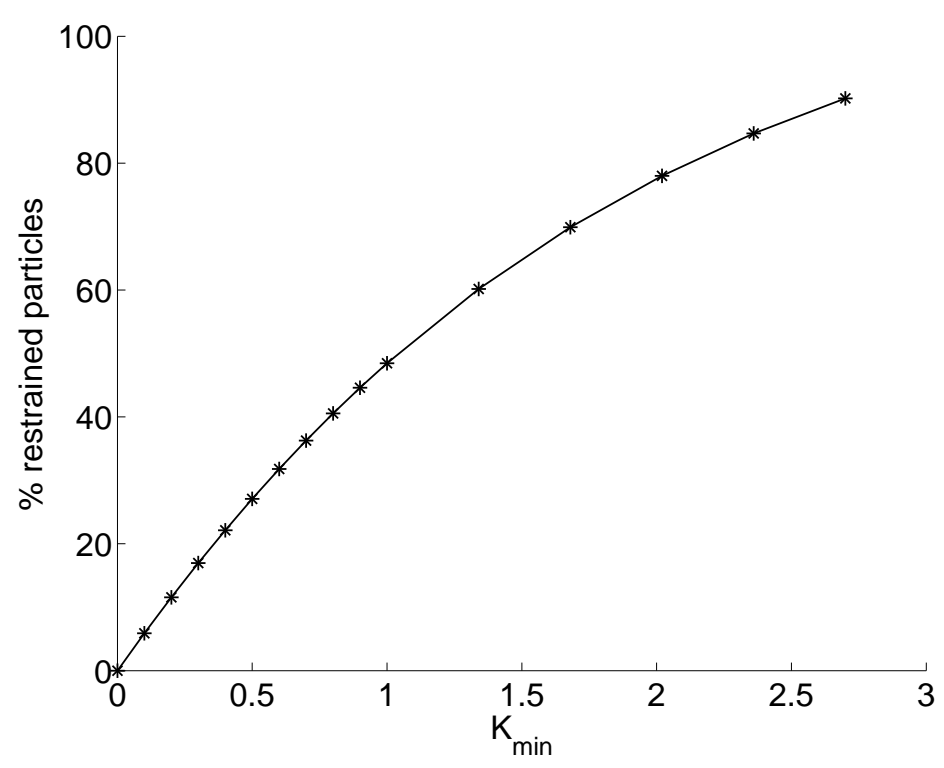

Fig. 17: Percentage of restrained particles as a function of $K_{\min }$.

The following result, central in this proof, gives estimates on derivatives of $u(t)$ in the weighted spaces $L^{2}\left(\pi_{s}\right)$ (see Section 6.3.2 for the proof).

Lemma 4 Suppose that Assumptions 2 and 5 hold. For any $n \geqslant 1$, there exists $\lambda_{n}>0$ and $s_{n} \in \mathbb{N}$ such that, for $s \geqslant s_{n}$ and $G_{v} \leqslant \rho_{s}$ with $\rho_{s}$ sufficiently small, there is $r \in \mathbb{N}$ and $C>0$ for which

$$
\forall|k| \leqslant n, \quad \int_{\mathscr{E}}\left|\partial^{k} u(t, q, p)\right|^{2} \pi_{S}(p) d p d q \leqslant C\|A\|_{W_{\mathscr{K}_{r}}^{n, \infty}}^{2} \exp (-\lambda t) .
$$

Assume in the sequel that $G_{v} \leqslant \rho_{s}$ for $s$ sufficiently large. In view of the estimates (28), and using the fact that $\partial^{j} \pi_{s}(p)=\psi_{j, s}(p) \pi_{s}(p)$ with $\psi_{j, s}(p) \rightarrow 0$ as $|p| \rightarrow+\infty$, we obtain that, for any $n \geqslant 1$, there exist $s_{n} \in \mathbb{N}$ such that, for $s \geqslant s_{n}$, it is possible to find $r \in \mathbb{N}$ and $C>0$ for which

$\forall|k|+|\ell| \leqslant n, \quad \forall t \geqslant 0, \quad \int_{\mathscr{E}}\left|\partial^{\ell}\left(\partial^{k} u(t, q, p) \pi_{s}(p)\right)\right|^{2} d p d q \leqslant C\|A\|_{W_{\varkappa_{\varkappa_{r}}}^{\tilde{n} \infty \infty}}^{2} \exp (-\lambda t)$.

By the Sobolev embedding theorem, we can conclude that, for any $n \geqslant 1$, there exist $s_{n}, \widetilde{n} \in \mathbb{N}$ such that, for $s \geqslant s_{n}$ and provided $G_{v} \leqslant \rho_{s}$, it is possible to find $r \in \mathbb{N}$ and $C>0$ for which

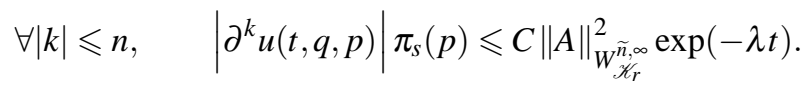

This concludes the proof of Lemma 3. 


\subsubsection{Proof of Lemma 4}

The main tool in the proof of Lemma 4 is the following estimate, which is the counterpart of (Kopec 2013, Lemma A.6) for our modified Langevin dynamics.

Lemma 5 Let $\mathscr{A}$ be a linear operator. Assume that $U \in \mathscr{S}$ and $\Delta U \in L^{\infty}$. There exists an integer $s_{*}$ such that, for all $s \geqslant s_{*}$, there is a constant $\omega_{s}>0$ for which the following inequality holds true for any $\zeta, T>0$ :

$$
\begin{aligned}
& \exp (\zeta T) \int_{\mathscr{E}}|\mathscr{A} u(t)|^{2} \pi_{s} d q d p+\frac{2 \gamma}{\beta} \int_{0}^{T} \exp (\zeta t)\left(\int_{\mathscr{E}}\left|\nabla_{p} \mathscr{A} u(t)\right|^{2} \pi_{s} d q d p\right) d t \\
& \leqslant \int_{\mathscr{E}}|\mathscr{A} u(0)|^{2} \pi_{s} d q d p+\left(\omega_{s}+\gamma\|\Delta U\|_{L^{\infty}}+\zeta\right) \int_{0}^{T} \exp (\zeta t)\left(\int_{\mathscr{E}}|\mathscr{A} u(t)|^{2} \pi_{s} d p d q\right) d t \\
& \quad+2 \int_{0}^{T} \exp (\zeta t)\left(\int_{\mathscr{E}}[\mathscr{A}, \mathscr{L}] u(t) \mathscr{A} u(t) \pi_{s} d q d p\right) d t
\end{aligned}
$$

In fact, a careful inspection of the proof shows that, since $U \in \mathscr{S}$, it is possible to avoid the assumption $\Delta U \in L^{\infty}$ by appropriately increasing the Lyapunov index $s$. Since $\Delta U \in L^{\infty}$ for AR-Langevin dynamics, we however keep this assumption.

Proof A simple computation shows that

$$
2 \mathscr{L} \mathscr{A} u(t) \mathscr{A} u(t)=\mathscr{L}\left(|\mathscr{A} u(t)|^{2}\right)-\frac{2 \gamma}{\beta}\left|\nabla_{p} \mathscr{A} u(t)\right|^{2}
$$

The formal adjoint of the operator $\mathscr{L}$ in $L^{2}(\mathscr{E})$ is given by

$$
\mathscr{L}^{\dagger}=-\left(\nabla U \cdot \nabla_{q}-\nabla V \cdot \nabla_{p}\right)+\gamma\left(\Delta U+\nabla U \cdot \nabla_{p}+\frac{1}{\beta} \Delta_{p}\right) .
$$

In view of Assumption 2, there exists therefore $\omega_{s}>0$ such that

$$
\begin{aligned}
\mathscr{L}^{\dagger} \pi_{s} & =\left[-(\nabla V+\gamma \nabla U) \cdot \frac{\nabla \mathscr{K}_{s}}{\mathscr{K}_{s}}+\gamma \Delta U-\frac{\gamma}{\beta} \frac{\Delta \mathscr{K}_{s}}{\mathscr{K}_{s}}+\frac{2 \gamma}{\beta} \frac{\left|\nabla \mathscr{K}_{s}\right|^{2}}{\mathscr{K}_{s}^{2}}\right] \pi_{s} \\
& \leqslant\left(\omega_{s}+\gamma\|\Delta U\|_{L^{\infty}}\right) \pi_{s} .
\end{aligned}
$$

With this estimation, we can follow exactly the proof of (Kopec 2013, Lemma A.6), i.e. write the expression for $\frac{d}{d t}\left[\exp (\zeta t)|\mathscr{A} u(t)|^{2}\right]$, use (30), integrate the resulting expression in time and with respect to $\pi_{s} d p d q$ (for $s$ sufficiently large), and finally use (31) to deduce (29).

Let us now prove Lemma 4. The complete proof is done by induction on $n$. We provide here the complete proofs for $n=0$ and $n=1$, and only sketch the extension to higher orders of derivation since the proof follows the same lines as in (Kopec 2013, Appendix A). 
Case $n=0$. Recall first that, in view of Assumption 2, the exponential convergence of the law provided by Theorem 4 holds. Denote by $\lambda_{\ell}$ the corresponding exponential rate of decay for a given $\ell \in \mathbb{N}^{*}$. For any $r \in \mathbb{N}$, we directly obtain the following decay estimates in $L^{2}\left(\pi_{l}\right)$ when $l>2 r+d / 2$ : there exists $\widetilde{C}_{l, r}>0$ such that

$$
\int_{\mathscr{E}}|u(t)|^{2} \pi_{l} \leqslant \widetilde{C}_{l, r} \mathrm{e}^{-2 \lambda_{r} t}\|A\|_{L_{\mathscr{K}_{r}}^{\infty}}^{2} .
$$

Note that this corresponds to the case $n=0$ in Lemma 4 .

Case $n=1$. We now prove the estimates in the case $n=1$. We first apply Lemma 5 with $\mathscr{A}=$ Id: there exists $s_{*} \in \mathbb{N}$ such that, for all $s \geqslant s_{*}$ and $\zeta<2 \lambda_{r}$, there is $C>0$ and $r \in \mathbb{N}$ for which

$$
\forall T \in \mathbb{R}_{+}, \quad \int_{0}^{T} \exp (\zeta t)\left[\int_{\mathscr{E}}\left|\nabla_{p} u(t, q, p)\right|^{2} \pi_{s}(p) d q d p\right] d t \leqslant C\|A\|_{L_{\mathscr{K}_{r}}^{\infty}}^{2} .
$$

In order to control derivatives in $q$, the key idea, going back to Talay (2002), is to use mixed derivatives $\alpha \partial_{p_{i}}-\partial_{q_{i}}$ (for some parameter $\alpha>0$ ). This allows indeed to retrieve some dissipation in the $q$ direction when $\nabla^{2} U$ is positive definite. The next lemma is the most important part of our proof since we show how to extend the use of mixed derivatives to the case when $\nabla^{2} U$ is not positive definite.

Lemma 6 Consider the operator $L_{\alpha}:=\alpha \nabla_{p}-\nabla_{q}$ for some parameter $\alpha \in \mathbb{R}$. There exists $s_{*} \in \mathbb{N}$ such that, for $s \geqslant s_{*}$ and provided $G_{v} \leqslant \rho_{s}$ (for some constant $\rho_{s}>0$ defined in (36) below), there is $r \in \mathbb{N}, \zeta<2 \lambda_{r}, \alpha>0$ and $C>0$ for which

$\forall T>0, \int_{0}^{T} \exp (\zeta t)\left[\int_{\mathscr{E}}\left(\left|L_{\alpha} u(t, q, p)\right|^{2}+\left|\nabla_{p} L_{\alpha} u(t, q, p)\right|^{2}\right) \pi_{s}(p) d q d p\right] d t \leqslant C\|A\|_{W_{\mathscr{K}_{r}}^{1, \infty}}^{2}$.

Proof Define $L_{\alpha, i}:=\alpha \partial_{p_{i}}-\partial_{q_{i}}$ for $i \in\{1, \ldots, d\}$. The commutator of $L_{\alpha, i}$ and $\mathscr{L}$ is

$$
\begin{aligned}
{\left[L_{\alpha, i}, \mathscr{L}\right] } & =-\alpha\left(\nabla_{p} \partial_{p_{i}} U\right) \cdot\left(\gamma \nabla_{p}-\nabla_{q}\right)+\left(\nabla_{q} \partial_{q_{i}} V\right) \cdot \nabla_{p} \\
& =-\alpha\left(\nabla_{p} \partial_{p_{i}} U\right) \cdot L_{\alpha}-\alpha(\gamma-\alpha)\left(\nabla_{p} \partial_{p_{i}} U\right) \cdot \nabla_{p}+\left(\nabla_{q} \partial_{q_{i}} V\right) \cdot \nabla_{p} .
\end{aligned}
$$

Introducing $C_{V}:=\sup _{i, j=1, \ldots, d}\left\|\partial_{q_{i} q_{j}}^{2} V\right\|_{L^{\infty}}$, a simple computation shows that

$$
\begin{aligned}
& 2 \sum_{i=1}^{d} L_{\alpha, i} u(t)\left[L_{\alpha, i}, \mathscr{L}\right] u(t)= \\
& =-2 \alpha \sum_{i=1}^{d} \sum_{j=1}^{d} L_{i} u(t)\left(\partial_{p_{j}} \partial_{p_{i}} U\right) L_{\alpha, j} u(t) \\
& \quad+2 \alpha(\alpha-\gamma) \sum_{i=1}^{d} L_{\alpha, i} u(t)\left(\nabla \partial_{p_{i}} U\right) \cdot \nabla_{p} u(t)+2 \sum_{i=1}^{d} L_{\alpha, i} u(t) \nabla\left(\partial_{q_{i}} V\right) \cdot \nabla_{p} u(t) \\
& \leqslant \\
& \quad\left(\varepsilon_{1}+\varepsilon_{2}-2 v \alpha\right)\left|L_{\alpha} u(t)\right|^{2}-2 \alpha \sum_{i=1}^{d} \sum_{j=1}^{d} L_{\alpha, i} u(t) \partial_{p_{j}} \partial_{p_{i}}\left(U-U_{v}\right) L_{\alpha, j} u(t) \\
& \quad+\frac{\alpha^{2}(\gamma-\alpha)^{2}}{\varepsilon_{1}}\left(\sup _{|j|=2}\left\|\partial^{j} U\right\|_{L^{\infty}}^{2}\right)\left|\nabla_{p} u(t)\right|^{2}+\frac{C_{V}^{2}}{\varepsilon_{2}}\left|\nabla_{p} u(t)\right|^{2}
\end{aligned}
$$


for any $\varepsilon_{1}, \varepsilon_{2}>0$. With this preliminary computation, we can now choose $\mathscr{A}=L_{\alpha, i}$ in Lemma 5 and sum over $i=1, \ldots, d$ : for $s \geqslant s_{*}$ with $s_{*}$ sufficiently large,

$$
\begin{aligned}
& \exp (\zeta T) \int_{\mathscr{E}}\left|L_{\alpha} u(t)\right|^{2} \pi_{s} d q d p+\frac{2 \gamma}{\beta} \int_{0}^{T} \exp (\zeta t)\left(\int_{\mathscr{E}} \sum_{i=1}^{d}\left|\nabla_{p} L_{\alpha, i} u(t)\right|^{2} \pi_{s} d q d p\right) d t \\
& \quad \leqslant \int_{\mathscr{E}}\left|L_{\alpha} u(0)\right|^{2} \pi_{s} d q d p \\
& \quad+\left(\omega_{s}+\gamma\|\Delta U\|_{L^{\infty}}^{2}+\zeta+\varepsilon_{1}+\varepsilon_{2}-2 v \alpha\right) \int_{0}^{T} \exp (\zeta t)\left(\int_{\mathscr{E}}\left|L_{\alpha} u(t)\right|^{2} \pi_{s} d q d p\right) d t \\
& \quad-2 \alpha \int_{0}^{T} \exp (\zeta t)\left(\int_{\mathscr{E}} L_{\alpha} u(t)^{T}\left[\nabla^{2}\left(U-U_{v}\right)\right] L_{\alpha} u(t) \pi_{s} d q d p\right) d t \\
& \quad+\frac{\alpha^{2}(\gamma-\alpha)^{2}}{\varepsilon_{1}}\left(\sup _{|j|=2}\left\|\partial^{j} U\right\|_{L^{\infty}}^{2}\right) \int_{0}^{T} \exp (\zeta t)\left(\int_{\mathscr{E}}\left|\nabla_{p} u(t)\right|^{2} \pi_{s} d q d p\right) d t \\
& \quad+\frac{C_{V}^{2}}{\varepsilon_{2}} \int_{0}^{T} \exp (\zeta t)\left(\int_{\mathscr{E}}\left|\nabla_{p} u(t)\right|^{2} \pi_{s} d q d p\right) d t .
\end{aligned}
$$

Since $L_{\alpha} u(0)=\left(\alpha \nabla_{p}-\nabla_{q}\right) A \in \widetilde{\mathscr{W}}_{\mathscr{K}_{r}}^{1, \infty}$ for some integer $r \leqslant s_{*}$ (upon increasing $s_{*}$ ), and in view of (32), the first and the two last terms of the right hand side of the above inequality can be controlled uniformly in time for $\zeta<2 \lambda_{r}$.

It remains to take care of the terms (34) and (35). Our strategy is to prove that they are negative when $\zeta<2 \lambda_{r}$, and can hence be transfered to the lef-hand side of the inequality. To simplify the notation, we denote $\widetilde{U}:=U-U_{v}$. Recall that, by Assumption 12, it holds $\|\nabla \widetilde{U}\|_{L^{\infty}} \leqslant G_{v}$. An integration by parts shows that

$$
\begin{aligned}
& -\int_{\mathscr{E}} \sum_{j=1}^{d} L_{\alpha, i} u(t)\left(\partial_{p_{j}} \partial_{p_{i}} \widetilde{U}\right) L_{\alpha, j} u(t) \pi_{s} d q d p=\int_{\mathscr{E}}\left(\nabla \widetilde{U} \cdot L_{\alpha} u(t)\right) \operatorname{div}_{p}\left(L_{\alpha} u(t)\right) \pi_{s} d q d p \\
& \quad+\int_{\mathscr{E}} \sum_{j=1}^{d}\left(\partial_{p_{i}} \widetilde{U}\right) L_{\alpha, j} u(t)\left(\partial_{p_{j}} L_{\alpha, i} u(t)\right) \pi_{s} d q d p+\int_{\mathscr{E}} \sum_{j=1}^{d}\left(\nabla \widetilde{U} \cdot L_{\alpha} u(t)\right)\left(\nabla \pi_{s} \cdot L_{\alpha} u(t)\right) d q d p .
\end{aligned}
$$

With this expression we now estimate the term (35) by

$$
\begin{aligned}
&-2 \alpha \int_{0}^{T} \exp (\zeta t)\left(\int_{\mathscr{E}} \sum_{i=1}^{d} \sum_{j=1}^{d} L_{i} u(t)\left(\partial_{p_{j}} \partial_{p_{i}} \widetilde{U}\right) L_{j} u(t) \pi_{s} d q d p\right) d t \\
& \leqslant 2\left(1+\mathscr{G}_{S}\right) \alpha \varepsilon_{3}\|\nabla \widetilde{U}\|_{L^{\infty}} \int_{0}^{T} \exp (\zeta t)\left(\int_{\mathscr{E}}|L u(t)|^{2} \pi_{s} d q d p\right) d t \\
&+\frac{2 \alpha}{\varepsilon_{3}}\|\nabla \widetilde{U}\|_{L^{\infty}} \int_{0}^{T} \exp (\zeta t)\left(\int_{\mathscr{E}} \sum_{i=1}^{d}\left|\nabla_{p} L_{i} u(t)\right|^{2} \pi_{s} d q d p\right) d t
\end{aligned}
$$

where we have used Young's inequality and introduced a constant $\mathscr{G}_{s} \in \mathbb{R}_{+}$such that $\left|\nabla_{p} \pi_{s}\right| \leqslant \mathscr{G}_{s} \pi_{s}$ 
The following conditions are therefore sufficient to ensure that (34) and (35) are non-positive when $\zeta<2 \lambda_{r}$ : there exists $\alpha>0$ such that

$$
\omega_{s}+\gamma\|\Delta U\|_{L^{\infty}}^{2}+\zeta-2 v \alpha+2 \alpha \varepsilon_{3}\left(\mathscr{G}_{s}+1\right)\|\nabla \widetilde{U}\|_{L^{\infty}}<0
$$

and

$$
\frac{2 \gamma}{\beta}>\frac{2 \alpha}{\varepsilon_{3}}\|\nabla \widetilde{U}\|_{L^{\infty}}
$$

These conditions can be restated as

$$
\frac{\omega_{s}+\gamma\|\Delta U\|_{L^{\infty}}^{2}+\zeta}{2\left(v-\varepsilon_{3}\left(\mathscr{G}_{s}+1\right)\|\nabla \widetilde{U}\|_{L^{\infty}}\right)}<\alpha<\frac{\gamma \varepsilon_{3}}{\beta\|\nabla \widetilde{U}\|_{L^{\infty}}} .
$$

Since $\zeta$ can be chosen arbitrarily small (while still being positive), the latter condition holds provided $\|\nabla \widetilde{U}\|_{L^{\infty}}$ :

$$
\|\nabla \widetilde{U}\|_{L^{\infty}}<\frac{2 \gamma v \varepsilon_{3}}{\beta\left(\omega_{s}+\gamma\|\Delta U\|_{L^{\infty}}^{2}+\zeta\right)+2 \gamma\left(\mathscr{G}_{s}+1\right) \varepsilon_{3}^{2}} .
$$

After optimization with respect to $\varepsilon_{3}$, this leads to the final condition

$$
\|\nabla \widetilde{U}\|_{L^{\infty}}<\sqrt{\frac{v^{2} \gamma}{2 \beta\left(\omega_{s}+\gamma\|\Delta U\|_{L^{\infty}}^{2}+\zeta\right)\left(\mathscr{G}_{S}+1\right)}} .
$$

In conclusion, defining

$$
\rho_{s}=\sqrt{\frac{v^{2} \gamma}{2 \beta\left(\omega_{s}+\gamma\|\Delta U\|_{L^{\infty}}^{2}\right)\left(\mathscr{G}_{s}+1\right)}},
$$

we see that the estimate (33) holds when the constant $G_{V}$ from Assumption 5 satisfies $G_{V} \leqslant \rho_{s}$.

The remainder of the proof of Lemma 4 is very similar to the corresponding proof in Kopec (2013). We first combine (32) and Lemma 6: there exists $s_{*} \in \mathbb{N}$ such that for $s \geqslant s_{*}$ there exists an integer $r$, a sufficiently small $\zeta<2 \lambda_{r}$ and $\rho_{s}>0$ such that if $G_{v} \leqslant \rho_{s}$, then there is a constant $C>0$ for which

$$
\forall T \geqslant 0, \quad \int_{0}^{T} \exp (\zeta t)\left(\int_{\mathscr{E}}\left|\nabla_{q} u(t)\right|^{2} \pi_{s} d q d p\right) d t \leqslant C\|A\|_{W_{\mathscr{K}_{r}}^{1, \infty}}^{2} .
$$

We can now again apply Lemma 5 , and sum the estimates obtained with $\mathscr{A}=\partial_{p_{i}}$. Before stating the result, we bound the integrand of the term involving the commutator $\left[\partial_{p_{i}}, \mathscr{L}\right]($ for $i=1, \ldots, d)$ as:

$$
\begin{aligned}
\left|\sum_{i=1}^{d}\left[\partial_{p_{i}}, \mathscr{L}\right] u(t) \partial_{p_{i}} u(t)\right| & =\left|\sum_{i=1}^{d} \nabla_{p}\left(\partial_{p_{i}} U\right) \cdot\left(\nabla_{q}-\gamma \nabla_{p}\right) u(t) \partial_{p_{i}} u(t)\right| \\
& \leqslant d\left(\sup _{|j|=2}\left\|\partial^{j} U\right\|_{L^{\infty}}^{2}\right)\left|\left(\nabla_{q}-\gamma \nabla_{p}\right) u(t)\right|\left|\nabla_{p} u(t)\right| \\
& \leqslant d\left(\sup _{|j|=2}\left\|\partial^{j} U\right\|_{L^{\infty}}^{2}\right)\left[\frac{1}{2}\left|\nabla_{q} u(t)\right|^{2}+\left(\gamma+\frac{1}{2}\right)\left|\nabla_{p} u(t)\right|^{2}\right] .
\end{aligned}
$$


Then, for $s \geqslant s_{*}$ (with $s_{*}$ sufficiently large) and for all $T \geqslant 0$,

$$
\begin{aligned}
& \exp (\zeta T) \int_{\mathscr{E}}\left|\nabla_{p} u(t)\right|^{2} \pi_{s} d q d p \\
& \leqslant \int_{\mathscr{E}}\left|\nabla_{p} u(0)\right|^{2} \pi_{s} d q d p+d\left(\sup _{|j|=2}\left\|\partial^{j} U\right\|_{L^{\infty}}\right) \int_{0}^{T} \exp (\zeta t)\left(\int_{\mathscr{E}}\left|\nabla_{q} u(t)\right|^{2} \pi_{s} d q d p\right) d t \\
& \quad+\left(\omega_{s}+\zeta+\sup _{|j|=2}\left\|\partial^{j} U\right\|_{L^{\infty}}(d+2 \gamma d+\gamma)\right) \int_{0}^{T} \exp (\zeta t)\left(\int_{\mathscr{E}}\left|\nabla_{p} u(t)\right|^{2} \pi_{s} d q d p\right) d t
\end{aligned}
$$

In view of (32) and (37) and since $\nabla_{p} u(0)=\nabla_{p} A \in \widetilde{W}_{\mathscr{K}_{\tilde{r}}}^{1, \infty}$ for some integer $\widetilde{r} \in \mathbb{N}$, we see that there exists $s_{*} \geqslant 1$ sufficiently large such that, for any $s \geqslant s_{*}$ and $\zeta>0$ sufficiently small, and provided $G_{V} \leqslant \rho_{s}$, there is a constant $C>0$ and an integer $r$ for which

$$
\int_{\mathscr{E}}\left|\nabla_{p} u(t)\right|^{2} \pi_{s} d q d p \leqslant C\|A\|_{W_{\mathscr{K}_{r}}^{1, \infty}}^{2} \exp (-\zeta T)
$$

To conclude to Lemma 4 for $n=1$, it remains to apply Lemma 5 with $\mathscr{A}=\nabla_{q}$ in order to obtain an estimate similar to the one above, but for $\left|\nabla_{q} u(t)\right|^{2}$. This is possible in view of the following bounds on the commutator: for all $i=1, \ldots, d$,

$$
\left|\left[\partial_{q_{i}}, \mathscr{L}\right] u(t) \partial_{q_{i}} u(t)\right|=\left|\nabla\left(\partial_{q_{i}} V\right) \cdot \nabla_{p} u(t) \partial_{q_{i}} u(t)\right| \leqslant\left|\nabla_{p} u(t)\right|^{2}+C_{V}^{2}\left|\partial_{q_{i}} u(t)\right|^{2}
$$

General $n$. The remainder of the proof is done by induction of $n$ and relies on the control of the commutators $\left[\partial_{q}^{k}, \mathscr{L}\right]$ with $|k|=n$, which are independent of $U$, as well as

$$
\left|\left[\partial_{p}^{k}, \mathscr{L}\right] \psi\right| \leqslant \sum_{\substack{i \in \mathbb{N}^{2 d} \\|i| \leqslant n}} \mathscr{P}_{i}\left|\partial^{i} \psi\right|
$$

where $\mathscr{P}_{i}$ are positive polynomial functions that depend on the polynomial growth of $U$ and its derivatives. These polynomial functions can be controlled with Lyapunov weights for sufficiently large indices. In addition, the same approach as in the proof of Lemma 6 is used to estimate the extra term arising from missing positivity of $\nabla^{2} U$, namely

$$
\begin{aligned}
-2 \alpha & \int_{0}^{T} \exp (\zeta t)\left(\int_{\mathscr{E}} \sum_{i=1}^{d} \sum_{j=1}^{d} L_{\alpha, i}\left(\partial_{q}^{n} u(t)\right)\left[\partial_{p_{i} p_{j}}^{2}\left(U-U_{v}\right)\right] L_{\alpha, j}\left(\partial_{q}^{n} u(t)\right) \pi_{s} d q d p\right) d t \\
\leqslant & 2\left(1+\mathscr{G}_{S}\right) \alpha \varepsilon_{3}\|\nabla \widetilde{U}\|_{L^{\infty}} \int_{0}^{T} \exp (\zeta t)\left(\int_{\mathscr{E}}\left|L_{\alpha}\left(\partial_{q}^{n} u(t)\right)\right|^{2} \pi_{s} d q d p\right) d t \\
& +\frac{2 \alpha}{\varepsilon_{3}}\|\nabla \widetilde{U}\|_{L^{\infty}} \int_{0}^{T} \exp (\zeta t)\left(\int_{\mathscr{E}}\left|\nabla_{p} L_{\alpha}\left(\partial_{q}^{n} u(t)\right)\right|^{2} \pi_{s} d q d p\right) d t
\end{aligned}
$$

Therefore, the result is obtained when the same condition (36) on $G_{V}$ is satisfied. Note however that this condition depends on $s$, hence on $n$ since $s$ has to be larger than some index $s_{n}$. 


\subsection{Proof of Proposition 1}

\subsubsection{General structure of the proof}

We define the AR perturbation function as

$$
D_{K_{\min }}(p):=\nabla U_{0, K_{\max }}(p)-\nabla U_{K_{\min }, K_{\max }}(p) .
$$

This allows to write the generator $\mathscr{L}_{K_{\min }, K_{\max }}$ of the AR-Langevin dynamics (6) as a perturbation of the generator $\mathscr{L}_{0, K_{\max }}$ :

$$
\mathscr{L}_{K_{\min }, K_{\max }}=\mathscr{L}_{0, K_{\max }}-D_{K_{\min }}(p) \cdot \tilde{\mathscr{L}}, \quad \tilde{\mathscr{L}}:=\nabla_{q}-\gamma \nabla_{p}
$$

For notational convenience we omit the subscript $K_{\max }$ and simply write $\mathscr{L}_{K_{\min }}:=$ $\mathscr{L}_{K_{\min }, K_{\max }}$. We also denote by $\mu_{K_{\min }}$ the invariant measure associated with $\mathscr{L}_{K_{\min }}$, and by $\Pi_{K_{\min }}$ the projection

$$
\Pi_{K_{\min }} f=f-\int_{\mathscr{E}} f d \mu_{K_{\min }} .
$$

For a given observable $A \in \mathscr{S}$, the asymptotic variance associated with the corresponding time averages reads, in view of (16):

$$
\sigma_{A}^{2}\left(K_{\min }\right)=-2 \int_{\mathscr{E}} \Phi_{A, K_{\min }} A d \mu_{K_{\min }}
$$

where $\Phi_{A, K_{\text {min }}} \in \mathscr{S}$ is the unique solution in $L_{\mathscr{K}_{s}}^{\infty}$ ( $s$ being such that $\left.A \in L_{\mathscr{K}_{s}}^{\infty}\right)$ of the following Poisson equation:

$$
\mathscr{L}_{K_{\min }} \Phi_{A, K_{\min }}=\Pi_{K_{\min }} A, \quad \Pi_{K_{\min }} \Phi_{A, K_{\min }}=0 .
$$

Similarly, the limiting variance for $K_{\min }=0$ can be rewritten as

$$
\sigma_{A}^{2}(0)=-2 \int_{\mathscr{E}} \Phi_{A, 0} A d \mu_{0}, \quad \mathscr{L}_{0} \Phi_{A, 0}=\Pi_{0} A, \quad \Pi_{0} \Phi_{A, 0}=0
$$

In order to prove the convergence of (38) to (40) and to identify the linear term in $K_{\min }$, the idea is to expand $\mu_{K_{\min }}$ and $\Phi_{A, K_{\min }}$ in powers of $K_{\min }$. To this end, we rewrite the Poisson equation (39) as

$$
\Pi_{0}\left(\Pi_{0}-\mathscr{L}_{0}^{-1} \Pi_{0} D_{K_{\min }} \cdot \tilde{\mathscr{L}}\right) \Phi_{A, K_{\min }}=\mathscr{L}_{0}^{-1} \Pi_{0} A
$$

The operator $\mathscr{L}_{0}^{-1} \Pi_{0} D_{K_{\min }} \cdot \tilde{\mathscr{L}}$ is not bounded (since $\tilde{\mathscr{L}}$ contains derivatives in $q$, which cannot be controlled by $\mathscr{L}_{0}$ ), so that it is not possible to write the inverse of $\Pi_{0}-\mathscr{L}_{0}^{-1} \Pi_{0} D_{K_{\min }} \cdot \tilde{\mathscr{L}}$ as some Neumann series. It is however possible to consider a pseudo-inverse operator by truncating the Neumann series at order $n$. This motivates the introduction of the following approximation of the solution of (39):

$$
\Phi_{A, K_{\min }}^{n}:=\sum_{k=0}^{n}\left(\mathscr{L}_{0}^{-1} \Pi_{0} D_{K_{\min }} \cdot \tilde{\mathscr{L}}\right)^{k} \mathscr{L}_{0}^{-1} \Pi_{0} A
$$

The corresponding approximation of the variance reads

$$
\sigma_{A, n}^{2}\left(K_{\min }\right):=-2 \int_{\mathscr{E}}\left(\Pi_{K_{\min }} \Phi_{A, K_{\min }}^{n}\right) A d \mu_{K_{\min }} .
$$


The connection with the exact variance (38) is given by the following lemma, which is proved in Section 6.4.5. We introduce a critical value $K_{\max }^{*}$ such that Assumption 5 is satisfied for $0 \leqslant K_{\min } \leqslant K_{\max } / 2$ and $K_{\max } \leqslant K_{\max }^{*}$ (see Section 6.4.3). This allows to resort to Lemma 3.

Lemma 7 Fix $0<K_{\max } \leqslant K_{\max }^{*}$. Then, for any $A \in \mathscr{S}$ and for all $n \geqslant 1$, there exists a constant $C_{A, n}>0$ such that

$$
\forall 0 \leqslant K_{\min } \leqslant \frac{K_{\max }}{2}, \quad\left|\sigma_{A}^{2}\left(K_{\min }\right)-\sigma_{A, n}^{2}\left(K_{\min }\right)\right| \leqslant C_{A, n} K_{\min }^{n+1} .
$$

The key point in the proof of Lemma 7 are the following estimates (see Section 6.4.4 for the proof).

Lemma 8 Fix $0<K_{\max } \leqslant K_{\max }^{*}$ and $A \in \mathscr{S}$. For any $n \geqslant 1$, there exist $s_{n}, l_{n} \in \mathbb{N}$ such that, for any $s \geqslant s_{n}$, there is $r_{n} \in \mathbb{N}$ and $\tilde{C}_{n}>0$ for which

$$
\forall 0 \leqslant K_{\min } \leqslant \frac{K_{\max }}{2}, \quad\left\|\left(\mathscr{L}_{0}^{-1} \Pi_{0} D_{K_{\min }} \cdot \tilde{\mathscr{L}}\right)^{n} \Pi_{0} A\right\|_{L_{\mathscr{K}_{s}}^{\infty}} \leqslant \tilde{C}_{n} K_{\min }^{n}\|A\|_{W_{\mathscr{K}_{n}}}^{l_{n, \infty}} .
$$

Proposition 1 now straightforwardly follows by combining Lemma 7 and the following expansion in powers of $K_{\min }$ of the truncated variance (whose proof can be read in Section 6.4.6).

Proposition 2 Fix $0<K_{\max } \leqslant K_{\max }^{*}$. There exists a constant $\mathscr{K} \in \mathbb{R}$ such that, for any $n \geqslant 1$ and $0 \leqslant K_{\min } \leqslant K_{\max } / 2$ sufficiently small,

$$
\sigma_{A, n}^{2}\left(K_{\text {min }}\right)=\sigma_{A}^{2}(0)+\mathscr{K} K_{\text {min }}+\mathrm{O}\left(K_{\text {min }}^{2}\right) .
$$

\subsubsection{Technical results on expansions with respect to $K_{\min }$}

Recall that the function $f_{0, K_{\max }}$ (with $f_{0, K_{\max }}$ defined in (17)) belongs to $C^{\infty}(\mathbb{R},[0,1])$. The next result shows that the same is true for

$$
f_{K_{\min }, K_{\max }}=f_{0, K_{\max }} \circ \theta_{K_{\min }},
$$

with $\theta_{K_{\min }}$ defined in (18). This is not obvious a priori since $\theta_{K_{\min }}$ is only piecewise $C^{\infty}$, with singularities on the first order derivative at $K_{\min }$ and $K_{\max }$. In fact, it can even be proved that $f_{K_{\min }, K_{\max }}-f_{0, K_{\max }}$ and all its derivatives are small when $K_{\min }$ is small.

Lemma 9 For any $0 \leqslant K_{\min }<K_{\max }$, the function $f_{K_{\min }, K_{\max }}$ belongs to $C^{\infty}(\mathbb{R},[0,1])$. Moreover, its derivatives have a compact support in $\left[0, K_{\max }\right]$. Finally, for any $n_{0} \in \mathbb{N}$ and $\delta>0$, there exists a constant $C_{n_{0}, K_{\max }, \delta}>0$ such that

$\forall 0 \leqslant n \leqslant n_{0}, \quad \forall K_{\min } \in\left[0, K_{\max }-\delta\right], \quad\left\|f_{K_{\min }, K_{\max }}^{(n)}-f_{0, K_{\max }}^{(n)}\right\|_{L^{\infty}} \leqslant C_{n_{0}, K_{\max }, \delta} K_{\min }$. 
Proof The function $\theta_{K_{\min }}$ is defined piecewise on three intervals $\left[0, K_{\min }\right),\left(K_{\min }, K_{\max }\right)$ and $\left(K_{\max },+\infty\right)$. In the interior of each interval, both $f_{0, K_{\max }}$ and $\theta_{K_{\min }}$ are $C^{\infty}$, and so is therefore their composition. In addition, $f_{K_{\min }, K_{\max }}$ is constant on $\left(K_{\max },+\infty\right)$, hence all derivatives vanish on this interval. To prove that $f_{K_{\min }, K_{\max }}$ is $C^{\infty}$ with derivatives of compact support, it therefore suffices to prove that all derivatives can be extended by continuity at the points $K_{\min }$ and $K_{\max }$.

Since $f_{0, K_{\max }}$ is constant outside the interval $\left[K_{\min }, K_{\max }\right]$, a simple computation shows that, for $n \geqslant 1$,

$$
\left(f_{0, K_{\max }} \circ \theta_{K_{\min }}\right)^{(n)}(x)=\left\{\begin{aligned}
0 & \text { for } 0 \leqslant x<K_{\min }, \\
\left(\frac{K_{\max }}{K_{\max }-K_{\min }}\right)^{n} f_{0, K_{\max }}^{(n)}\left(\theta_{K_{\min }}(x)\right) & \text { for } K_{\min }<x<K_{\max }, \\
0 & \text { for } x>K_{\max } .
\end{aligned}\right.
$$

It is therefore obvious to check the continuity at $K_{\min }$ and $K_{\max }$ since all derivatives of $f_{0, K_{\max }}$ vanish at 0 and $K_{\max }$, and $\theta_{K_{\min }}\left(K_{\min }\right)=0$ while $\theta_{K_{\min }}\left(K_{\max }\right)=K_{\max }$.

Moreover, it is easy to check that $\left|\theta_{K_{\min }}(x)-x\right| \leqslant K_{\min }$, so that the estimate (42) already follows in the case $n=0$ since $f_{0, K_{\max }}$ is Lipschitz continuous. To obtain the same result for higher order derivatives, we note that the $n$-th order derivative can be rewritten as

$$
f_{K_{\min }, K_{\max }}^{(n)}=f_{0, K_{\max }}^{(n)} \circ \theta_{K_{\min }}+f_{0, K_{\max }}^{(n)} \circ \theta_{K_{\min }}\left(\left[\left(\frac{K_{\max }}{K_{\max }-K_{\min }}\right)^{n}-1\right] \mathbb{1}_{\left[K_{\min }, K_{\max }\right]}\right) .
$$

Therefore, $f_{K_{\min }, K_{\max }}^{(n)}-f_{0, K_{\max }}^{(n)}$ is the sum of (i) $f_{0, K_{\max }}^{(n)}\left(\theta_{K_{\min }}\right)-f_{0, K_{\max }}^{(n)}$, which is of order $K_{\min }$ in $L^{\infty}$ norm by the same argument as before since $f_{0, K_{\max }}^{(n)}$ is Lipschitz continuous; and (ii) a remainder term of order $K_{\min }$ since $f_{0, K_{\max }}^{(n)} \circ \theta_{K_{\min }}$ is uniformly bounded; while for any $0<\delta<K_{\max }$ there exists $R_{n, \delta}>0$ such that

$$
\forall K_{\min } \in\left[0, K_{\max }-\delta\right], \quad\left|\left(\frac{K_{\max }}{K_{\max }-K_{\min }}\right)^{n}-1\right| \leqslant R_{n, \delta} K_{\min } .
$$

This allows to obtain the desired result.

In view of the definition (19)-(20) of $U_{K_{\min }, K_{\max }}(p)=\sum_{i=1}^{N} u_{K_{\min }, K_{\max }}\left(p_{i}\right)$, we can deduce the following estimates on $U_{K_{\min }, K_{\max }}-U_{0, K_{\max }}$ and its derivatives, which allow in particular to control $D_{K_{\min }}$. To state the result, we introduce

$$
\mathscr{C}_{K}:=\left\{p \in \mathbb{R}^{d} \mid \forall i=1, \ldots N, \frac{p_{i}^{2}}{2 m_{i}} \leqslant K\right\} .
$$

Corollary 2 For any $0 \leqslant K_{\min }<K_{\max }$, the function $U_{K_{\min }, K_{\max }}$ belongs to $C^{\infty}$. For any $n \geqslant 0$ and $|\alpha|=n$, the function $\partial^{\alpha}\left(U_{K_{\min }, K_{\max }}-U_{0, K_{\max }}\right)$ has a compact support in $\mathscr{C}_{K_{\max }}$. Moreover, for any $n_{0} \geqslant 0$ and $\delta>0$, there exists a constant $C_{n_{0}, \delta, K_{\max }}>0$ such that

$\forall|\alpha| \leqslant n_{0}, \forall K_{\min } \in\left[0, K_{\max }-\delta\right], \quad\left\|\partial^{\alpha} U_{K_{\min }, K_{\max }}-\partial^{\alpha} U_{0, K_{\max }}\right\|_{L^{\infty}} \leqslant C_{n_{0}, \delta, K_{\max }} K_{\min }$. 
In order to obtain more precise statements about the behavior of the functions $f_{K_{\min }, K_{\max }}(x)$ for small values of $K_{\min }$, a natural idea would be to perform Taylor expansions with respect to this parameter. The difficulty is however that the derivatives with respect to $K_{\min }$ of the shift function $\theta_{K_{\min }}(x)$ are not continuous in $x$. This prevents to write directly remainders of order $K_{\min }^{2}$. Before stating the precise result in Lemma 11, we need another technical ingredient.

Lemma 10 Fix $K_{\max }>0$ and define $\tilde{\tau}(x):=\frac{x-K_{\max }}{K_{\max }}$. Then, for any $n \geqslant 0$ and $\delta>0$, there exists $C_{n, \delta}>0$ such that

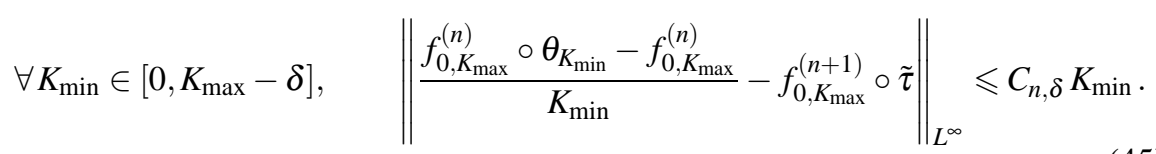

Proof Note that, formally, $\tilde{\tau}$ is the derivative of $\theta_{K_{\min }}$ on $\left[K_{\min }, K_{\max }\right]$ with respect to $K_{\min }$, evaluated at $K_{\min }=0$. Recall also $\left|\theta_{K_{\min }}(x)-x\right| \leqslant K_{\min }$. Simple computations show that there exists $C_{\delta}>0$ such that

$$
\forall x \in \mathbb{R}^{+}, \quad\left|\frac{\theta_{K_{\min }}(x)-x}{K_{\min }}-\tilde{\tau}(x)\right| \leqslant C_{\delta} K_{\min } .
$$

Since $f_{0, K_{\min }} \in C^{\infty}$, there exists $t \in[0,1]$ such that

$$
f_{0, K_{\max }}^{(n)}\left(\theta_{K_{\min }}(x)\right)-f_{0, K_{\max }}^{(n)}(x)=f_{0, K_{\max }}^{(n+1)}\left(x+t\left(\theta_{K_{\min }}(x)-x\right)\right)\left(\theta_{K_{\min }}(x)-x\right) .
$$

Therefore, for $x \in\left[0, K_{\max }\right]$,

$$
\begin{aligned}
& \left|\frac{f_{0, K_{\max }}^{(n)}\left(\theta_{K_{\min }}(x)\right)-f_{0, K_{\max }}^{(n)}(x)}{K_{\min }}-f_{0, K_{\max }}^{(n+1)}(x) \tilde{\tau}(x)\right| \\
& =\left|f_{0, K_{\max }}^{(n+1)}\left(x+t\left(\theta_{K_{\min }}(x)-x\right)\right) \frac{\theta_{K_{\min }}(x)-x}{K_{\min }}-f_{0, K_{\max }}^{(n+1)}(x) \tilde{\tau}(x)\right| \\
& \leqslant\left|f_{0, K_{\max }}^{(n+1)}\left(x+t\left(\theta_{K_{\min }}(x)-x\right)\right)-f_{0, K_{\max }}^{(n+1)}(x)\right|\left|\frac{\theta_{K_{\min }}(x)-x}{K_{\min }}\right|+\left|f_{0, K_{\max }}^{(n+1)}(x)\right|\left|\frac{\theta_{K_{\min }}(x)-x}{K_{\min }}-\tilde{\tau}(x)\right| \\
& \leqslant\left(\left\|f_{0, K_{\max }}^{(n+2)}\right\|_{L^{\infty}\left(\left[0, K_{\max }\right]\right)}+C_{\delta}\left\|f_{0, K_{\max }}^{(n+1)}\right\|_{L^{\infty}\left(\left[0, K_{\max }\right]\right)}\right) K_{\min },
\end{aligned}
$$

where we have used the following equality: there exists $\alpha \in[0,1]$ such that

$f_{0, K_{\max }}^{(n+1)}\left(x+t\left(\theta_{K_{\min }}(x)-x\right)\right)-f_{0, K_{\max }}^{(n+1)}(x)=t f_{0, K_{\max }}^{(n+2)}\left(x+\alpha\left(\theta_{K_{\min }}(x)-x\right)\right)\left(\theta_{K_{\min }}(x)-x\right)$,

together with the bound $\left|\theta_{K_{\min }}(x)-x\right| \leqslant K_{\min }$. 
Lemma 11 Fix $K_{\max }>0$. There exist functions $\mathscr{D}_{i} \in C^{\infty}\left(\mathbb{R}^{d}\right)($ for $i=1, \ldots, N)$, with compact support in $\mathscr{C}_{K_{\max }}$, such that, for $0<\delta<K_{\max }$ and $r \in \mathbb{N}$, there is $C_{r, \delta}>0$ such that

$$
K_{\min } \in\left[0, K_{\max }-\delta\right], \quad\left\|D_{K_{\min }, i}-K_{\min } \mathscr{D}_{i}\right\|_{W^{r, \infty}} \leqslant C_{r, \delta} K_{\min }^{2}
$$

Proof Recall that the functions $D_{K_{\min }, i}: \mathbb{R}^{D} \rightarrow \mathbb{R}^{D}$ are defined, for $i=1, \ldots, N$, as

$$
\begin{aligned}
D_{K_{\min }, i}(p)= & {\left[f_{K_{\min }, K_{\max }}\left(\frac{\left|p_{i}\right|^{2}}{2 m_{i}}\right)-f_{0, K_{\max }}\left(\frac{\left|p_{i}\right|^{2}}{2 m_{i}}\right)\right] \frac{p_{i}}{m_{i}} } \\
& +\frac{\left|p_{i}\right|^{2}}{2 m_{i}}\left[f_{K_{\min }, K_{\max }}^{\prime}\left(\frac{\left|p_{i}\right|^{2}}{2 m_{i}}\right)-f_{0, K_{\max }}^{\prime}\left(\frac{\left|p_{i}\right|^{2}}{2 m_{i}}\right)\right] \frac{p_{i}}{m_{i}} .
\end{aligned}
$$

We next define, for $i=1, \ldots, N$, the function

$$
\mathscr{D}_{i}(p):=\left\{\begin{aligned}
{\left[f_{0, K_{\max }}^{\prime}\left(\frac{\left|p_{i}\right|^{2}}{2 m_{i}}\right)+\frac{\left|p_{i}\right|^{2}}{2 m_{i}} f_{0, K_{\max }}^{\prime \prime}\left(\frac{\left|p_{i}\right|^{2}}{2 m_{i}}\right)\right] \tilde{\tau}\left(\frac{\left|p_{i}\right|^{2}}{2 m_{i}}\right) \frac{p_{i}}{m_{i}}, } & \text { for } \frac{\left|p_{i}\right|^{2}}{2 m_{i}} \in\left[0, K_{\max }\right], \\
0, & \text { for } \frac{\left|p_{i}\right|^{2}}{2 m_{i}} \geqslant K_{\max },
\end{aligned}\right.
$$

where $\tilde{\tau}$ is defined in Lemma 10. Recall that $f_{0, K_{\max }} \in C^{\infty}$ and $f_{0, K_{\max }}^{(n)}$ have compact support on $\left[0, K_{\max }\right]$ for $n \geqslant 1$. Therefore, $\mathscr{D}_{i} \in C^{\infty}$ also has compact support in $\mathscr{C}_{K_{\max }}$.

The case $r=0$ of (48) follows directly from Lemma 10 with $n=0$ and $n=1$. Let us now consider the case $r=1$ more carefully. To simplify the presentation, we consider separately the two terms in the sums defining the functions $D_{K_{\min }, i}$ and $\mathscr{D}_{i}$, i.e. $D_{K_{\min }, i}=D_{K_{\min }, i, 1}+D_{K_{\min }, i, 2}$ and $\mathscr{D}_{i}=\mathscr{D}_{i, 1}+\mathscr{D}_{i, 2}$ with

$$
D_{K_{\min }, i, 1}(p)=\left[f_{K_{\min }, K_{\max }}\left(\frac{\left|p_{i}\right|^{2}}{2 m_{i}}\right)-f_{0, K_{\max }}\left(\frac{\left|p_{i}\right|^{2}}{2 m_{i}}\right)\right] \frac{p_{i}}{m_{i}}
$$

and

$$
\mathscr{D}_{i, 1}(p):=\left\{\begin{aligned}
f_{0, K_{\max }}^{\prime}\left(\frac{\left|p_{i}\right|^{2}}{2 m_{i}}\right) \tilde{\tau}\left(\frac{\left|p_{i}\right|^{2}}{2 m_{i}}\right) \frac{p_{i}}{m_{i}}, & \text { for } \frac{\left|p_{i}\right|^{2}}{2 m_{i}} \in\left[0, K_{\max }\right], \\
0, & \text { for } \frac{\left|p_{i}\right|^{2}}{2 m_{i}} \geqslant K_{\max }
\end{aligned}\right.
$$

We present the estimates only for the difference $D_{K_{\min }, i, 1} / K_{\min }-\mathscr{D}_{i, 1}$ since similar computations allows to control the difference $D_{K_{\min }, i, 2} / K_{\min }-\mathscr{D}_{i, 2}$. For $\alpha, \alpha^{\prime} \in$ $\{1, \ldots, D\}$, we denote by $p_{i, \alpha}$ the $\alpha$ th component of the momentum of the $i$ th particle 
and by $D_{K_{\min }, i, 1, \alpha^{\prime}}$ and $\mathscr{D}_{i, 1, \alpha^{\prime}}$ the $\alpha^{\prime}$ th components of $D_{K_{\min }, i, 1}$ and $\mathscr{D}_{i, 1}$. Then, for $p_{i} \in \mathscr{C}_{K_{\max }}$,

$$
\begin{aligned}
& \left|\partial_{p_{i, \alpha}}\left(\frac{D_{K_{\min }, i, 1, \alpha^{\prime}}}{K_{\min }}-\mathscr{D}_{i, 1, \alpha^{\prime}}\right)(p)\right|= \\
& \leqslant \frac{\delta_{\alpha, \alpha^{\prime}}}{m_{i}}\left|\frac{f_{0, K_{\max }} \circ \theta_{K_{\min }}\left(\frac{\left|p_{i}\right|^{2}}{2 m_{i}}\right)-f_{0, K_{\max }}\left(\frac{\left|p_{i}\right|^{2}}{2 m_{i}}\right)}{K_{\min }}-f_{0, K_{\max }}^{\prime}\left(\frac{\left|p_{i}\right|^{2}}{2 m_{i}}\right) \tilde{\tau}\left(\frac{\left|p_{i}\right|^{2}}{2 m_{i}}\right)\right| \\
& +\frac{\left|p_{i, \alpha} p_{i, \alpha^{\prime}}\right|}{m_{i}^{2}} \mid \frac{f_{0, K_{\max }}^{\prime} \circ \theta_{K_{\min }}\left(\frac{\left|p_{i}\right|^{2}}{2 m_{i}}\right) \theta_{K_{\min }}^{\prime}\left(\frac{\left|p_{i}\right|^{2}}{2 m_{i}}\right)-f_{0, K_{\max }}^{\prime}\left(\frac{\left|p_{i}\right|^{2}}{2 m_{i}}\right)}{K_{\min }} \\
& -f_{0, K_{\max }}^{\prime \prime}\left(\frac{\left|p_{i}\right|^{2}}{2 m_{i}}\right) \tilde{\tau}\left(\frac{\left|p_{i}\right|^{2}}{2 m_{i}}\right)-f_{0, K_{\max }}^{\prime}\left(\frac{\left|p_{i}\right|^{2}}{2 m_{i}}\right) \frac{1}{K_{\max }} \\
& \leqslant \frac{\delta_{\alpha, \alpha^{\prime}}}{m_{i}}\left|\frac{f_{0, K_{\max }} \circ \theta_{K_{\min }}\left(\frac{\left|p_{i}\right|^{2}}{2 m_{i}}\right)-f_{0, K_{\max }}\left(\frac{\left|p_{i}\right|^{2}}{2 m_{i}}\right)}{K_{\min }}-f_{0, K_{\max }}^{\prime}\left(\frac{\left|p_{i}\right|^{2}}{2 m_{i}}\right) \tilde{\tau}\left(\frac{\left|p_{i}\right|^{2}}{2 m_{i}}\right)\right| \\
& +\frac{\left|p_{i, \alpha} p_{i, \alpha^{\prime}}\right|}{m_{i}^{2}}\left|\frac{f_{0, K_{\max }}^{\prime} \circ \theta_{K_{\min }}\left(\frac{\left|p_{i}\right|^{2}}{2 m_{i}}\right)-f_{0, K_{\max }}^{\prime}\left(\frac{\left|p_{i}\right|^{2}}{2 m_{i}}\right)}{K_{\min }}-f_{0, K_{\max }}^{\prime \prime}\left(\frac{\left|p_{i}\right|^{2}}{2 m_{i}}\right) \tilde{\tau}\left(\frac{\left|p_{i}\right|^{2}}{2 m_{i}}\right)\right| \\
& +\frac{\left|p_{i, \alpha} p_{i, \alpha^{\prime}}\right|}{m_{i}^{2}}\left|\left[f_{0, K_{\max }}^{\prime} \circ \theta_{K_{\min }}\left(\frac{\left|p_{i}\right|^{2}}{2 m_{i}}\right)-f_{0, K_{\max }}^{\prime}\left(\frac{\left|p_{i}\right|^{2}}{2 m_{i}}\right)\right] \frac{\theta_{K_{\min }}^{\prime}\left(\frac{\left|p_{i}\right|^{2}}{2 m_{i}}\right)-1}{K_{\min }}\right| \\
& +\frac{\left|p_{i, \alpha} p_{i, \alpha^{\prime}}\right|}{m_{i}^{2}}\left|f_{0, K_{\max }}^{\prime}\left(\frac{\left|p_{i}\right|^{2}}{2 m_{i}}\right)\left[\frac{\theta_{K_{\min }}^{\prime}\left(\frac{\left|p_{i}\right|^{2}}{2 m_{i}}\right)-1}{K_{\min }}-\frac{1}{K_{\max }}\right]\right|
\end{aligned}
$$

where we used $\tilde{\tau}^{\prime}(x)=1 / K_{\max }$ for $x \in\left[0, K_{\max }\right]$. The first two terms in the last inequality can be bounded by $C^{*} K_{\min }$ for some constant $C^{*} \in \mathbb{R}_{+}$in view of Lemma 10 . 
For the last two terms, distinguish the cases $p_{i} \in \mathscr{C}_{i, K_{\min }}$ and $p_{i} \in \mathscr{C}_{i, K_{\max }} \backslash \mathscr{C}_{i, K_{\min }}$, where for $K \geqslant 0$ we define

$$
\mathscr{C}_{i, K}:=\left\{p_{i} \in \mathbb{R}^{D} \mid \frac{\left|p_{i}\right|^{2}}{2 m_{i}} \leqslant K\right\} .
$$

When $p_{i} \in \mathscr{C}_{i, K_{\min }}$, the third term disappears since $\theta_{K_{\min }}^{\prime}(x)=1$ on $\left[0, K_{\min }\right]$. In addition,

$$
\sup _{p_{i} \in \mathscr{C}_{i, K_{\min }}} \frac{\left|p_{i}\right|^{2}}{m_{i}^{2}} \leqslant \frac{2 K_{\min }}{m_{i}}
$$

so that

$$
\left\|\partial_{p_{i, \alpha}}\left(\frac{D_{K_{\min }, i, 1, \alpha^{\prime}}}{K_{\min }}-\mathscr{D}_{i, 1, \alpha^{\prime}}\right)\right\|_{L^{\infty}\left(\mathscr{C}_{i, K_{\min }}\right)} \leqslant\left(C^{*}+\frac{2}{m_{i} K_{\max }}\left\|f_{0, K_{\max }}^{\prime}\right\|_{L^{\infty}\left(\left[0, K_{\min }\right]\right)}\right) K_{\min } .
$$

When $p_{i} \in \mathscr{C}_{i, K_{\max }} \backslash \mathscr{C}_{i, K_{\min }}$, we use $\theta_{K_{\min }}^{\prime}(x)=K_{\max } /\left(K_{\max }-K_{\min }\right)$ for $x \in\left[K_{\min }, K_{\max }\right]$, so that there exists $C_{\delta}>0$ such that

$$
\sup _{x \in\left[K_{\min }, K_{\max }\right]}\left|\frac{\theta_{K_{\min }}^{\prime}(x)-1}{K_{\min }}-\frac{1}{K_{\max }}\right| \leqslant C_{\delta} K_{\min }, \quad \sup _{x \in\left[K_{\min }, K_{\max }\right]}\left|\theta_{K_{\min }}^{\prime}(x)-1\right| \leqslant \frac{1}{K_{\max }-K_{\min }} \leqslant \frac{1}{\delta} .
$$

Using these bounds as well as the inequality $\left|\theta_{K_{\min }}(x)-x\right| \leqslant K_{\min }$ and (46) for $n=1$, it follows

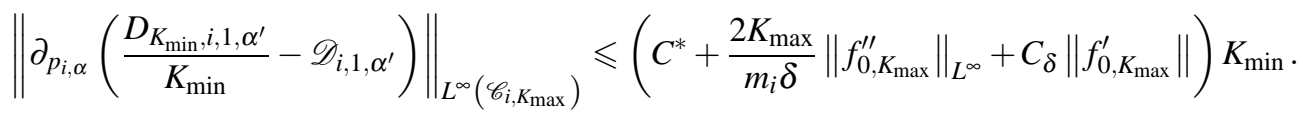

This concludes the proof of (48) for $r=1$.

Bounds on higher order derivatives are obtained in a similar fashion, relying on the fact that $\partial_{x}^{2} \theta_{K_{\min }}(x)=0$ except at the singularity points $K_{\min }, K_{\max }$ as well as $\partial_{x}^{2} \tilde{\tau}(x)=0$ for $x \neq K_{\max }$.

We end this section with a last technical result.

Lemma 12 Fix $K_{\max }>0$. Then for any $f \in L^{1}\left(\mu_{0}\right)$, there exist $a_{f} \in \mathbb{R}$ such that, for $0<\delta<K_{\max }$

$\forall K_{\min } \in\left[0, K_{\max }-\delta\right], \quad \int_{\mathbb{R}^{d}} f(p) \mathrm{e}^{-\beta U_{K_{\min }}(p)} d p=\int_{\mathbb{R}^{d}} f(p) \mathrm{e}^{-\beta U_{0}(p)} d p+a_{f} K_{\min }+\mathrm{O}\left(K_{\min }^{2}\right)$.

Proof Recall that $U_{K_{\min }}(p)=\sum_{i=1}^{N} u_{K_{\min }, K_{\max }}\left(p_{i}\right)$. Note that

$$
u_{K_{\min }, K_{\max }}\left(p_{i}\right)-u_{0, K_{\max }}\left(p_{i}\right)=\frac{p_{i}^{2}}{2 m_{i}}\left[f_{0, K_{\max }}\left(\frac{p_{i}^{2}}{2 m_{i}}\right)-f_{K_{\min }, K_{\max }}\left(\frac{p_{i}^{2}}{2 m_{i}}\right)\right] .
$$

Manipulations similar to the ones used to prove (48) allow to show that there exists a function $\mathscr{U} \in C^{\infty}$ with compact support in $\mathscr{C}_{K_{\max }}$ such that, for $0<\delta<K_{\max }$ and $r \in \mathbb{N}$, there is $C_{r, \delta}>0$ for which

$$
K_{\min } \in\left[0, K_{\max }-\delta\right], \quad\left\|U_{K_{\min }}-U_{0}-K_{\min } \mathscr{U}\right\|_{W^{r, \infty}} \leqslant C_{r, \delta} K_{\min }^{2} .
$$


This allows to write

$$
U_{K_{\min }}=U_{0}+K_{\min } \mathscr{U}+K_{\min }^{2} \widetilde{\mathscr{U}}_{K_{\min }}
$$

with $\widetilde{\mathscr{U}}_{K_{\min }}$ uniformly bounded in $L^{\infty}$. Moreover since $U_{K_{\min }}-U_{0} \in C^{\infty}$ also has a compact support, we easily obtain

$\frac{\mathrm{e}^{-\beta U_{K_{\min }}-\mathrm{e}^{-\beta U_{0}}}}{K_{\min }}=\mathrm{e}^{-\beta U_{0}} \frac{\mathrm{e}^{-\beta K_{\min }\left(\mathscr{U}+K_{\min } \tilde{\mathscr{U}}_{\min }\right)}-1}{K_{\min }}=-\beta \mathscr{U} \mathrm{e}^{-\beta U_{0}}+K_{\min } \widehat{\mathscr{U}}_{K_{\min }} \mathrm{e}^{-\beta U_{0}}$,

with $\widehat{\mathscr{U}}_{K_{\min }}$ uniformly bounded in $L^{\infty}$. Therefore, there exists a constant $R>0$ such that

$$
\left|\frac{\int_{\mathbb{R}^{d}} f \mathrm{e}^{-\beta U_{K_{\min }} d p-\int_{\mathbb{R}^{d}} f \mathrm{e}^{-\beta U_{0}} d p}}{K_{\min }}+\beta \int_{\mathbb{R}^{d}} f \mathscr{U} \mathrm{e}^{-\beta U_{0}} d p\right| \leqslant R K_{\min },
$$

so that (50) follows with $a_{f}:=-\beta \int_{\mathbb{R}^{d}} \mathscr{U} f \mathrm{e}^{-\beta U_{0}} d p$.

\subsubsection{Verification of Assumption 5}

In order to use Lemma 3, we need to check that Assumption 5 holds with $G_{V}$ as small as wanted for appropriate values of $K_{\min }, K_{\max }$. The first condition (10) is easy to check, so we concentrate on the last two conditions. The reference kinetic energy function $U_{v}$ in Lemma 3 is chosen as the standard kinetic energy $U_{\text {std }}(p)=$ $p^{T} M^{-1} p / 2$, so that $v=1 / \min _{i=1, \ldots, N}$. It therefore remains to check the last condition. An inspection of the proof of Lemma 3 reveals that it holds provided $K_{\min }, K_{\max }$ are such that (36) holds. Straightforward computations show that

$$
\nabla_{p_{i}}\left(U_{K_{\min }}-U_{\text {std }}\right)=\frac{p_{i}}{m_{i}}\left[1-f_{K_{\min }, K_{\max }}\left(\frac{p_{i}^{2}}{m_{i}}\right)\right]-\frac{p_{i}\left|p_{i}\right|^{2}}{m_{i}^{2}} f_{K_{\min }, K_{\max }}^{\prime}\left(\frac{p_{i}^{2}}{m_{i}}\right)
$$

so that, using the fact that $U_{K_{\min }, K_{\max }}-U_{\text {std }}$ has compact support in $\mathscr{C}_{K_{\max }}$ (hence $\left|p_{i}\right| \leqslant \sqrt{2 m_{i} K_{\max }}$ ) and in view of the expression (43) of $f_{K_{\min }, K_{\max }}^{\prime}$, the following bound holds:

$$
\left\|\nabla_{p_{i}}\left(U_{K_{\min }, K_{\max }}-U_{\text {std }}\right)\right\|_{L^{\infty}} \leqslant \sqrt{\frac{2 K_{\max }}{m_{i}}}+\sqrt{\frac{8 K_{\max }^{3}}{m_{i}}} \frac{K_{\max }}{K_{\max }-K_{\min }}\left\|f_{0, K_{\max }^{\prime}}^{\prime}\right\|_{L^{\infty}} .
$$

Similarly, there exists a constant $C>0$ (depending on $f_{0, K_{\max }}^{\prime}, f_{0, K_{\max }}^{\prime \prime}$ and $\left.m_{1}, \ldots, m_{N}\right)$ such that

$$
\left\|\Delta U_{K_{\min }, K_{\max }}\right\|_{L^{\infty}} \leqslant C\left[1+\left(\frac{K_{\max }^{2}}{K_{\max }-K_{\min }}\right)^{2}\right]
$$

It is then easy to see that (36) holds upon choosing $0<K_{\min } \leqslant K_{\max } / 2$ with $K_{\max }>0$ sufficiently small. 


\subsubsection{Proof of Lemma 8}

Denote by $\mathscr{A}$ the operator $\mathscr{L}_{0}^{-1} \Pi_{0}\left(D_{K_{\min }} \cdot \tilde{\mathscr{L}}\right)$. By Corollary 2 , for any $n \geqslant 0$ and $0 \leqslant K_{\min } \leqslant K_{\max } / 2$, there exists a constant $R_{n}>0$ such that

$$
\forall|\alpha| \leqslant n, \quad\left\|\partial_{p}^{\alpha} D_{K_{\min }}\right\|_{L^{\infty}} \leqslant R_{n} K_{\min } .
$$

By the resolvent estimate (9), there exists for any $s \in \mathbb{N}^{*}$ a constant $C_{s}>0$ such that

$$
\forall f \in \widetilde{L_{\mathscr{K}_{s}}^{\infty}}, \quad\left\|\mathscr{L}_{0}^{-1} f\right\|_{L_{\mathscr{K}_{s}}^{\infty}} \leqslant C_{s}\|f\|_{L_{\mathscr{K}_{s}}^{\infty}} .
$$

Therefore, choosing an integer $s$ for which $A \in W_{\mathscr{K}_{s}}^{1, \infty}$, there exists a constant $C>0$ such that

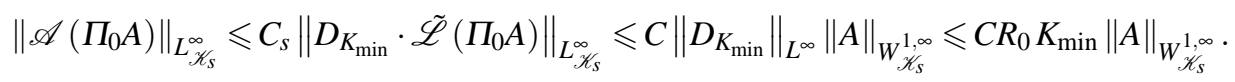

By the same principle, using the fact that, by (48), there is for any $r \geqslant 0$ a constant $C_{r}>0$ such that

$$
\left\|D_{K_{\min }}\right\|_{W^{r, \infty}} \leqslant C_{r} K_{\min }
$$

and in view of (15), there exists, for any $l \geqslant 0$, integers $\alpha \geqslant l$ and $s_{l} \in \mathbb{N}$ such that, for all $s \geqslant s_{l}$, there is a constant $C>0$ and an integer $r \in \mathbb{N}$ for which

$$
\left\|\mathscr{A}\left(\Pi_{0} A\right)\right\|_{W_{\mathscr{K}_{s}}^{l, \infty}} \leqslant C K_{\min }\|A\|_{W_{\mathscr{K}_{r}}^{\alpha, \infty}} .
$$

By recurrence, there exist, for any $n \geqslant 1$, integers $s_{n}, l_{n} \geqslant 0$ such that, for all $s \geqslant s_{n}$, there is $r \in \mathbb{N}$ and $\widetilde{C}>0$ for which

$$
\left\|\mathscr{A}^{n}\left(\Pi_{0} A\right)\right\|_{L_{\mathscr{K}_{s}}^{\infty}} \leqslant \widetilde{C} K_{\min }^{n}\|A\|_{W_{\mathscr{K}_{r}}^{l n, \infty}}
$$

This gives the claimed result.

\subsubsection{Proof of Lemma 7}

We start by writing the difference between the variance (38) and the truncated one (41):

$$
\sigma_{A}^{2}\left(K_{\min }\right)-\sigma_{A, n}^{2}\left(K_{\min }\right)=-2 \int\left(\Phi_{A, K_{\min }}-\Pi_{K_{\min }} \Phi_{A, K_{\min }}^{n}\right) A d \mu_{K_{\min }} .
$$

A simple computation gives

$$
\Pi_{K_{\min }} \mathscr{L}_{K_{\min }}\left(\Phi_{A, K_{\min }}-\Phi_{A, K_{\min }}^{n}\right)=-\Pi_{K_{\min }}\left(D_{K_{\min }} \cdot \tilde{\mathscr{L}}\right)\left(\mathscr{L}_{0}^{-1} \Pi_{0} D_{K_{\min }} \cdot \tilde{\mathscr{L}}\right)^{n} \mathscr{L}_{0}^{-1} \Pi_{0} A .
$$

We first use Lemma 8: there exists $s_{n}, l_{n} \in \mathbb{N}$ such that, for $s \geqslant s_{n}$, there is $r_{n} \in \mathbb{N}$ and $C>0$ such that

$$
\left\|\left(\mathscr{L}_{0}^{-1} \Pi_{0} D_{K_{\min }} \cdot \tilde{\mathscr{L}}\right)^{n} \mathscr{L}_{0}^{-1} \Pi_{0} A\right\|_{W_{\mathscr{K}_{s}}^{1, \infty}} \leqslant C K_{\min }^{n}\|A\|_{W_{\mathscr{K} r_{n}}^{l_{n}, \infty}} .
$$


Therefore, using (44) and (53), there exists some constant $R_{n}>0$ such that

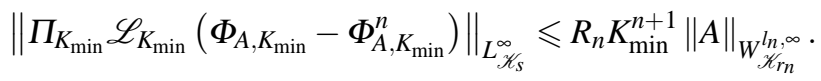

We finally apply $\mathscr{L}_{K_{\text {min }}}^{-1}$ to both sides of (53): in view of (14), it follows

$$
\left\|\Pi_{K_{\min }}\left(\Phi_{A, K_{\min }}-\Phi_{A, K_{\min }}^{n}\right)\right\|_{L_{\mathscr{K}_{s}}^{\infty}} \leqslant \frac{R_{n} C_{s}}{\lambda_{s}} K_{\min }^{n+1}\|A\|_{W_{\mathscr{K}_{n}}^{l_{n}, \infty}} .
$$

The result is then a direct consequence of the equality (52).

\subsubsection{Proof of Proposition 2}

Looking at (41), there are three objects which depend on the parameter $K_{\min }$ : the projection $\Pi_{K_{\min }}$, the truncated solution of the Poisson equation $\Phi_{A, K_{\min }}^{n}$ and the modified measure $\mu_{K_{\min }}$.

We first expand $\Phi_{A, K_{\min }}^{n}$ in terms of $\Phi_{A, 0}$ as

$$
\begin{aligned}
\Phi_{A, K_{\min }}^{n} & =\mathscr{L}_{0}^{-1} \Pi_{0} A+\left(\mathscr{L}_{0}^{-1} \Pi_{0} D_{K_{\min }} \cdot \tilde{\mathscr{L}}\right) \mathscr{L}_{0}^{-1} \Pi_{0} A+\sum_{k=2}^{n}\left(\mathscr{L}_{0}^{-1} \Pi_{0} D_{K_{\min }} \cdot \tilde{\mathscr{L}}\right)^{k} \mathscr{L}_{0}^{-1} \Pi_{0} A \\
& =\Phi_{A, 0}+\left(\mathscr{L}_{0}^{-1} \Pi_{0} D_{K_{\min }} \cdot \tilde{\mathscr{L}}\right) \Phi_{A, 0}+\sum_{k=2}^{n}\left(\mathscr{L}_{0}^{-1} \Pi_{0} D_{K_{\min }} \cdot \tilde{\mathscr{L}}\right)^{k} \Phi_{A, 0} \cdot
\end{aligned}
$$

Estimates on $\Phi_{A, 0}$ and its derivatives in terms of $A$ can be obtained with (15). Lemma 8 then allows to estimate the higher order terms in the above equality: there exists $s \in \mathbb{N}$ and $C>0$ such that

$$
\left\|\Phi_{A, K_{\min }}^{n}-\Phi_{A, 0}-\left(\mathscr{L}_{0}^{-1} \Pi_{0} D_{K_{\min }} \cdot \tilde{\mathscr{L}}\right) \Phi_{A, 0}\right\|_{L_{\mathscr{K}_{s}}^{\infty}} \leqslant C K_{\min }^{2}
$$

By combining these estimates with (48), we obtain

$$
\Phi_{A, K_{\min }}^{n}=\Phi_{A, 0}+K_{\min }\left(\mathscr{L}_{0}^{-1} \Pi_{0} \mathscr{D} \cdot \tilde{\mathscr{L}}\right) \Phi_{A, 0}+K_{\min }^{2} \mathscr{R}_{K_{\min }},
$$

where $\mathscr{R}_{K_{\min }}$ is uniformly bounded in $L_{\mathscr{K}_{s}}^{\infty}$ due to (48) for $K_{\min }$ small enough (upon possibly increasing $s$ ).

With the notation of Lemma 12 , for any $f \in L^{1}\left(\mu_{0}\right)$,

$$
\int_{\mathscr{E}} f d \mu_{K_{\min }}=\frac{\int_{\mathscr{E}} f \mathrm{e}^{-\beta U_{K_{\min }}}}{\int_{\mathscr{E}} \mathrm{e}^{-\beta U_{K_{\min }}}}=\int_{\mathscr{E}} f d \mu_{0}+\frac{a_{f}-a_{1} \int_{\mathscr{E}} f d \mu_{0}}{\int_{\mathscr{E}} \mathrm{e}^{-\beta U_{0}}} K_{\min }+\widetilde{\mathscr{R}}_{K_{\min }} K_{\min }^{2}
$$

with $\widetilde{\mathscr{R}}_{K_{\min }}$ uniformly bounded for $K_{\min }$ small enough. Finally, by combining (54) and (55), we see that there exists $\mathscr{K} \in \mathbb{R}$ such that

$$
\sigma_{A, n}^{2}\left(K_{\min }\right)=-2 \int_{\mathscr{E}} \Phi_{A, 0} A d \mu_{0}+\mathscr{K} K_{\min }+\mathrm{O}\left(K_{\min }^{2}\right) .
$$

Acknowledgements Stephane Redon and Zofia Trstanova gratefully acknowledge funding from the European Research Council through the ERC Starting Grant n. 307629. This work was funded by the Agence Nationale de la Recherche, under grant ANR-14-CE23-0012 (COSMOS). Gabriel Stoltz benefited from the scientific environment of the Laboratoire International Associé between the Centre National de la Recherche Scientifique and the University of Illinois at Urbana-Champaign. 


\section{References}

S. Artemova, S. Redon, Adaptively restrained particle simulations. Phys. Rev. Lett. 109(19), 190201 (2012)

C.H. Bennett, Mass tensor molecular dynamics. J. Comput. Phys. 19(3), 267-279 (1975)

R.N. Bhattacharya, On the functional Central Limit theorem and the law of the iterated logarithm for Markov processes. Z. Wahrscheinlichkeit. 60(2), 185-201 (1982)

M. Bosson, S. Grudinin, S. Redon, Block-adaptive quantum mechanics: an adaptive divide-and-conquer approach to interactive quantum chemistry. Journal of Computational Chemistry 34(6), 492-504 (2013)

M. Bosson, S. Grudinin, X. Bouju, S. Redon, Interactive physically-based structural modeling of hydrocarbon systems. Journal of Computational Physics 231(6), 2581-2598 (2012). doi:10.1016/j.jcp.2011.12.006

M. Hairer, J.C. Mattingly, Yet another look at Harris' ergodic theorem for Markov chains. Seminar on Stochastic Analysis, Random Fields and Applications VI 63, 109-117 (2011)

W.K. Hastings, Monte Carlo sampling methods using Markov chains and their applications. Biometrika 57, 97-109 (1970)

R. Joubaud, G.A. Pavliotis, G. Stoltz, Langevin dynamics with space-time periodic nonequilibrium forcing. J. Stat. Phys. 158(1), 1-36 (2015)

W. Kliemann, Recurrence and invariant measures for degenerate diffusions. Ann. Probab. 15(2), 690-707 (1987)

M. Kopec, Weak backward error analysis for Langevin process. arXiv preprint 1310.2599 (2013)

J.C. Latorre, G.A. Pavliotis, P.R. Kramer, Corrections to Einstein's relation for Brownian motion in a tilted periodic potential. J. Stat. Phys. 150(4), 776-803 (2013)

B. Leimkuhler, C. Matthews, G. Stoltz, The computation of averages from equilibrium and nonequilibrium Langevin molecular dynamics. IMA J. Numer. Anal. (2015)

T. Lelièvre, Two mathematical tools to analyze metastable stochastic processes, 791-810 (2013)

T. Lelièvre, G. Stoltz, Partial differential equations and stochastic methods in molecular dynamics. arXiv preprint (2015)

T. Lelièvre, M. Rousset, G. Stoltz, Free energy computations: A mathematical perspective (2010)

J.C. Mattingly, A.M. Stuart, D.J. Higham, Ergodicity for SDEs and approximations: locally Lipschitz vector fields and degenerate noise. Stoch. Proc. Appl. 101(2), 185-232 (2002)

N. Metropolis, A.W. Rosenbluth, M.N. Rosenbluth, A.H. Teller, E. Teller, Equations of state calculations by fast computing machines. J. Chem. Phys. 21(6), 1087-1091 (1953)

S.P. Meyn, R.L. Tweedie, Stability of Markovian processes. II. Continuous-time processes and sampled chains. Adv. Appl. Probab. 25, 487-517 (1993)

P. Plechac, M. Rousset, Implicit mass-matrix penalization of Hamiltonian dynamics with application to exact sampling of stiff systems. Multiscale Model. Sim. 8(2), 498-539 (2010)

L. Rey-Bellet, Ergodic properties of markov processes 1881, 1-39 (2006)

H. Risken, Fokker-planck equation (1984)

R. Rossi, M. Isorce, S. Morin, J. Flocard, K. Arumugam, S. Crouzy, M. Vivaudou, S. Redon, Adaptive torsion-angle quasi-statics: a general simulation method with applications to protein structure analysis and design. Bioinformatics 23(13) (2007). doi:10.1093/bioinformatics/btm191. http://bioinformatics.oxfordjournals.org/content/23/13/i408.abstract

G. Stoltz, Z. Trstanova, Numerical analysis of modified Langevin dynamics (In preparation)

J.E. Straub, M. Borkovec, B.J. Berne, Molecular-dynamics study of an isomerizing diatomic in a LennardJones fluid. J. Chem. Phys. 89(8), $4833-4847$ (1988)

D. Talay, Stochastic Hamiltonian dissipative systems: exponential convergence to the invariant measure, and discretization by the implicit Euler scheme. Markov Proc. Rel. Fields 8, 163-198 (2002)

M.E. Tuckerman, Statistical mechanics: Theory and molecular simulation (2010) 